\title{
Influence of Cross-Diffusion on Slip Flow and Heat Transfer of Chemically Reacting UCM Fluid between Porous Parallel Plates with Hall and Ion Slip Currents
}

\author{
Odelu Ojjela, Pravin Kashyap Kambhatla, N. Naresh Kumar, and Samir Kumar Das \\ Department of Applied Mathematics, Defence Institute of Advanced Technology (Deemed University), Pune 411025, India \\ Correspondence should be addressed to Odelu Ojjela; odelu3@yahoo.co.in
}

Received 9 March 2016; Revised 10 May 2016; Accepted 15 May 2016

Academic Editor: Clement Kleinstreuer

Copyright @ 2016 Odelu Ojjela et al. This is an open access article distributed under the Creative Commons Attribution License, which permits unrestricted use, distribution, and reproduction in any medium, provided the original work is properly cited.

\begin{abstract}
The present paper deals with the Hall and ion slip currents on an incompressible unsteady free convection flow and heat transfer of an upper convected Maxwell fluid between porous parallel plates with Soret and Dufour effects by considering the velocity slip and convective boundary conditions. Assume that there are periodic injection and suction at the lower and upper plates, respectively. The temperature and concentration at the lower and upper plates change periodically with time. The flow field equations are reduced to nonlinear ordinary differential equations by using similarity transformations and a semi-analytical-numerical solution has been obtained by the differential transform method. The velocity components, temperature distribution, and concentration with respect to different fluid and geometric parameters are discussed in detail and presented in the form of graphs. It is observed that the Biot number increases the temperature and concentration of the fluid. Further, the concentration of the fluid is enhanced whereas the temperature decreases with increasing slip. The present results are compared with the existing literature and are found to be in good agreement.
\end{abstract}

\section{Introduction}

The flow through porous channels is of great importance in both engineering and biological flows. Examples of this are found in aerodynamic heating, electrostatic precipitation, soil mechanics, food preservation, polymer technology, petroleum industry, the mechanics of the cochlea in the human ear, the flow of blood in the arteries, and artificial dialysis. The flows due to the periodic injection/suction at the boundary are of immense importance because of their wide range of applications. Several researchers have studied the incompressible laminar fluid flow between two porous parallel plates due to its mathematical simplicity and the flows can be analyzed easily in theoretical and experimental studies. Berman [1] examined the incompressible viscous fluid flow in a two-dimensional uniformly porous channel and a series solution was obtained for small values of the Reynolds number. Later, White Jr. et al. [2] considered the steady incompressible laminar viscous fluid flow between porous parallel plates with uniform suction or injection and the problem was analyzed for a wide range of suction Reynolds number. Walker and Davies [3] analyzed the mass transfer in an incompressible laminar viscous fluid flow between permeable parallel plates and a confluent hypergeometric function was used to obtain the solution. Hamza [4] investigated an incompressible laminar viscous fluid flow between two parallel rectangular as well as circular plates and obtained an approximate analytical solution by regular perturbation technique. The unsteady micropolar fluid flow between two parallel porous plates with periodic suction and injection was inspected by Srinivasacharya et al. [5]. The steady flow of chemically reacting micropolar fluid through a permeable channel was studied by Sheikholeslami et al. [6] and an analytical solution was obtained by using the homotopy perturbation method. Chen and Zhu [7] considered the slip effects on a Couette Poiseuille flow of a Bingham fluid between porous parallel plates and the reduced flow field equations are solved analytically. Singh and Pathak [8] analyzed the Hall currents and thermal radiation effects on unsteady MHD slip flow in a vertical rotating 
porous channel. Bhat and Mittal [9] inspected the Hall and ion slip currents on an electrically conducting viscous fluid flow through a parallel plate channel in the presence of uniform magnetic field and observed that the temperature of the fluid is reduced by Hall currents. Srinivasacharya and Kaladhar [10] examined analytically the Hall and ion slip currents on the mixed convective flow of couple stress fluid through a vertical parallel plate channel. Singh and Gorla [11] investigated the effects of thermal diffusion and heat source on free convection flow of a viscous fluid past an infinite vertical porous plate with Hall currents. Raptis et al. [12] studied the effects of Grashof number and permeability parameter on the free convection flow of a viscous fluid through a porous medium between two parallel plates. Abdulaziz and Hashim [13] investigated the heat and mass transfer of free convective flow of micropolar fluid in a vertical porous parallel plate channel and observed that the velocity decreases for increasing of Reynolds number. Makinde and Aziz [14] considered the convective boundary conditions for a MHD mixed convection fluid flow experiencing a firstorder chemical reaction over a vertical plate embedded in a porous medium and a numerical solution is obtained by using shooting technique along with a sixth-order RungeKutta integration. Yao et al. [15] analyzed an incompressible laminar viscous fluid flow and heat transfer over a stretching/shrinking sheet with convective boundary conditions and have found that the convective boundary condition results in a temperature slip at the wall. RamReddy et al. [16] studied an incompressible laminar free convective micropolar fluid flow along a permeable vertical plate with the convective boundary condition and obtained a numerical solution using spectral quasilinearization method. Postelnicu [17] examined the effects of Soret and Dufour on a two-dimensional steady stagnation point flow of a free convective Darcian fluid with suction/injection and the governing partial differential equations are reduced to nonlinear ordinary differential equations using similarity transformations and then solved by the Keller box method. Hayat et al. [18] investigated the threedimensional flow of a chemically reacting viscous fluid over exponentially stretching sheet with Soret and Dufour effects and observed that the thermal boundary layer increases for higher Dufour number while concentration boundary layer was increased for higher Soret values. Chamkha and Rashad [19] have numerically investigated the unsteady heat and mass transfer of an electrically conducting mixed convective chemically reacting viscous fluid flow over a rotating vertical cone with thermal-diffusion and diffusion-thermo effects. The MHD mixed convective heat and mass transfer of couple stress fluid through a porous channel with periodic suction and injection in the presence of cross-diffusion effects was considered by Ojjela and Naresh Kumar [20] and the reduced governing equations are solved numerically by the method of quasilinearization. The systematic framework of rate type viscoelastic fluids was developed by Oldroyd [21]. The unsteady three-dimensional flow of time dependent UCM fluid over a stretching sheet was studied by Awais et al. [22] and the series solution was obtained by HAM. Choi et al. [23] considered the two-dimensional steady incompressible laminar suction flow of a UCM fluid in a porous channel with the combined effects of inertia and viscoelasticity and obtained both analytical and numerical solutions. Hayat et al. [24] found an analytical solution for an electrically conducting Maxwell fluid peristaltic flow in a porous space. Abbas et al. [25] studied an incompressible magnetohydrodynamic two-dimensional boundary layer flow of UCM fluid through a rectangular porous channel using the homotopy analysis method. Mukhopadhyay and Gorla [26] analyzed the two-dimensional MHD flow and mass transfer of UCM fluid over an unsteady stretching sheet with first-order constructive/destructive chemical reaction and obtained a numerical solution by shooting method. Hayat and Abbas [27] inspected the effects of Deborah's number, Schmidt number, and chemical reaction parameter on velocity and concentration fields in the flow of chemically reacting UCM fluid between porous parallel plates. The unsteady MHD flow of Maxwell fluid over an impulsively stretching sheet was analyzed by Alizadeh-Pahlavan and Sadeghy [28] and the flow field equations are solved by the homotopy analysis method. Differential transform method is first used by Zhou [29] in solving circuit problems. The theory of two-dimensional differential transform method was proposed by Chen and Ho [30]. Ayaz [31] studied the solutions of differential equations using differential transform method in a three-dimensional platform. Recently, several researchers have obtained solutions for various problems in fluid mechanics by differential transform method due to its simplicity and high accuracy. Mosayebidorcheh et al. [32] have investigated the effect of mass transfer on an incompressible laminar upper convected Maxwell fluid in a porous channel with high permeability medium and the reduced flow field equations are solved by differential transform method. Sheikholeslami et al. [33] have considered an incompressible steady nanofluid flow and heat transfer between two horizontal parallel plates with thermophoresis and Brownian motion under the influence of uniform magnetic field and the governing equations are reduced into nondimensional ordinary differential equations using similarity transformation and then solved by differential transform method. Sheikholeslami and Ganji [34] have studied the effect of Brownian motion on a nanofluid in the presence of variable magnetic field and the solution is obtained by differential transform method. Hatami and Jing [35] have analyzed two different problems, the unsteady two-dimensional squeezing nanofluid flow and heat transfer between two parallel plates and a non-Newtonian free convective nanofluid flow, and heat transfer between two vertical flat plates and obtained a solution for both by differential transform method and the results are compared with RungeKutta fourth-order method. Esmail and Taha [36] have found a solution to the atmospheric dispersion equation by using DTM and the results are shown to be in good agreement with field data.

In the present paper, the effects of chemical reaction and Hall and ion slip currents on an unsteady incompressible free convective slip flow of an upper convected Maxwell fluid through a porous channel with Soret and Dufour in the presence of the convective boundary condition are considered. The reduced governing nonlinear ordinary differential equations are solved by differential transform method 


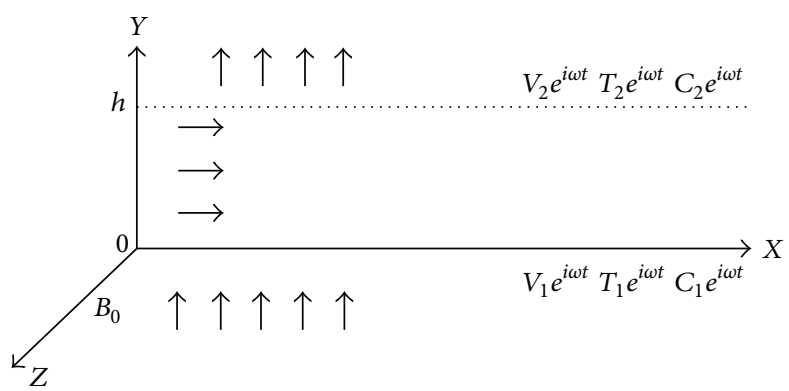

FIGURE 1: Schematic diagram of fluid flow between porous parallel plates.

(DTM). The effects of various fluid and geometric parameters on nondimensional velocity components, temperature distribution, and concentration are discussed in detail and shown in the form of graphs. The present results are compared with Bujurke et al. [37] for the Newtonian fluid and presented in the form of a table.

\section{Formulation of the Problem}

Consider an unsteady incompressible slip flow of an electrically conducting upper convected Maxwell fluid between two parallel plates which are separated by " $h$." Assume $V_{1} e^{i \omega t}$ and $V_{2} e^{i \omega t}$ are periodic injection and suction at the lower and upper plates, respectively. Also the temperature at the upper plate is maintained with $T_{2} e^{i \omega t}$, while the lower plate is governed by convective boundary condition and the concentrations at the lower and upper plates are $C_{1} e^{i \omega t}$ and $\mathrm{C}_{2} e^{i \omega t}$, respectively, as shown in Figure 1 .

The equations governing the heat and mass transfer of a UCM fluid flow in the presence of strong magnetic field and in the absence of body forces are $[22,28]$

$$
\begin{aligned}
& \frac{\partial u}{\partial x}+\frac{\partial v}{\partial y}=0 \\
& \rho\left[\frac{\partial u}{\partial t}+u \frac{\partial u}{\partial x}+v \frac{\partial u}{\partial y}\right. \\
& \left.+\beta\left(u^{2} \frac{\partial^{2} u}{\partial x^{2}}+v^{2} \frac{\partial^{2} u}{\partial y^{2}}+2 u v \frac{\partial^{2} u}{\partial x \partial y}\right)\right]=-\frac{\partial P}{\partial x} \\
& +\mu \nabla^{2} u-\sigma B_{0}^{2}\left(\frac{\left(1+\beta_{i} \beta_{e}\right) u-\beta_{e} v}{\left(1+\beta_{i} \beta_{e}\right)^{2}+\beta_{e}^{2}}\right)+\rho g \beta_{T}(T \\
& \rho\left[\frac{\partial v}{\partial t}+u \frac{\partial v}{\partial x}+v \frac{\partial v}{\partial y}\right. \\
& \left.+\beta\left(u^{2} \frac{\partial^{2} v}{\partial x^{2}}+v^{2} \frac{\partial^{2} v}{\partial y^{2}}+2 u v \frac{\partial^{2} v}{\partial x \partial y}\right)\right]=-\frac{\partial P}{\partial y} \\
& \quad+\mu \nabla^{2} v-\sigma B_{0}^{2}\left(\frac{\left(1+\beta_{i} \beta_{e}\right) v+\beta_{e} u}{\left(1+\beta_{i} \beta_{e}\right)^{2}+\beta_{e}^{2}}\right)
\end{aligned}
$$

$$
\begin{aligned}
& \rho c\left[\frac{\partial T}{\partial t}+u \frac{\partial T}{\partial x}+v \frac{\partial T}{\partial y}\right]=k\left(\frac{\partial^{2} T}{\partial x^{2}}+\frac{\partial^{2} T}{\partial y^{2}}\right) \\
& +\mu\left(2\left(\frac{\partial u}{\partial x}\right)^{2}+2\left(\frac{\partial v}{\partial y}\right)^{2}+\left(\frac{\partial u}{\partial y}+\frac{\partial v}{\partial x}\right)^{2}\right) \\
& +\sigma B_{0}^{2}\left(\frac{u^{2}+v^{2}}{\left(1+\beta_{i} \beta_{e}\right)^{2}+\beta_{e}^{2}}\right)+\frac{\rho D_{1} k_{T}}{c_{s}}\left(\frac{\partial^{2} C}{\partial x^{2}}\right. \\
& \left.+\frac{\partial^{2} C}{\partial y^{2}}\right), \\
& \rho c\left[\frac{\partial C}{\partial t}+u \frac{\partial C}{\partial x}+v \frac{\partial C}{\partial y}\right]=D_{1}\left(\frac{\partial^{2} C}{\partial x^{2}}+\frac{\partial^{2} C}{\partial y^{2}}\right) \\
& +\frac{D_{1} k_{T}}{T_{m}}\left(\frac{\partial^{2} T}{\partial x^{2}}+\frac{\partial^{2} T}{\partial y^{2}}\right)-k_{2}\left(C-C_{1} e^{i \omega t}\right) .
\end{aligned}
$$

The associated boundary conditions are as in (6)

$$
\begin{aligned}
u(x, \lambda, t) & =0, \\
v(x, \lambda, t) & =V_{1} e^{i \omega t}, \\
-k \frac{\partial T}{\partial y} & =h_{1}\left(T-T_{1} e^{i \omega t}\right), \\
C(x, \lambda, t) & =C_{1} e^{i \omega t} \\
u(x, \lambda, t) & =-\frac{\sqrt{k_{1}}}{\sigma_{1}} \frac{\partial u}{\partial y}, \\
v(x, \lambda, t) & =V_{2} e^{i \omega t}, \\
T(x, \lambda, t) & =T_{2} e^{i \omega t}, \\
C(x, \lambda, t) & =C_{2} e^{i \omega t}
\end{aligned}
$$


The governing equations are reduced to nondimensional equations using the following similarity transformations [20]:

$$
\begin{aligned}
& u(x, \lambda, t)=\left(\frac{U_{0}}{a}-\frac{V_{2} x}{h}\right) f^{\prime}(\lambda) e^{i \omega t}, \\
& v(x, \lambda, t)=V_{2} f(\lambda) e^{i \omega t}, \\
& T(x, \lambda, t) \\
& \quad=\left(T_{1}+\frac{\mu v_{2}}{\rho h c}\left(\phi_{1}(\lambda)+\left(\frac{U_{0}}{a V_{2}}-\frac{x}{h}\right)^{2} \phi_{2}(\lambda)\right)\right) e^{i \omega t},
\end{aligned}
$$

$$
C(x, \lambda, t)
$$

$$
=\left(C_{1}+\frac{\dot{n}_{A}}{h v}\left(g_{1}(\lambda)+\left(\frac{U_{0}}{a V_{2}}-\frac{x}{h}\right)^{2} g_{2}(\lambda)\right)\right) e^{i \omega t},
$$

where $f(\lambda), \phi_{1}(\lambda), \phi_{2}(\lambda), g_{1}(\lambda)$, and $g_{2}(\lambda)$ are unknown functions to be determined.

Substituting (7) in (2), (3), (4), and (5), we have

$$
\begin{aligned}
& f^{i v}\left(1-\mathrm{Wi} \operatorname{Re} f^{2} \cos \phi\right)=-\operatorname{Re} \cos \phi\left(f^{\prime} f^{\prime \prime}-f f^{\prime \prime \prime}\right) \\
& -2 \mathrm{Wi} \operatorname{Re}\left(f^{\prime 2} f^{\prime \prime}+f f^{\prime \prime 2}\right) \cos 2 \phi \\
& +\mathrm{Ha}^{2}\left(\frac{\left(1+\beta_{i} \beta_{e}\right)}{\left(1+\beta_{i} \beta_{e}\right)^{2}+\beta_{e}{ }^{2}}\right) f^{\prime \prime}-\frac{\mathrm{Ec} \mathrm{Gr}}{\xi}\left(\phi_{1}^{\prime}\right. \\
& \left.+\xi^{2} \phi_{2}^{\prime}\right)-\frac{\operatorname{Sh~Gc}}{\xi}\left(g_{1}^{\prime}+\xi^{2} g_{2}^{\prime}\right) \\
& \phi_{1}^{\prime \prime}=-\frac{\operatorname{Re} \operatorname{Pr} \cos \phi}{(1-\mathrm{DuScSr})}\left(4\left(f^{\prime}\right)^{2}\right. \\
& \left.+\left(\frac{\mathrm{Ha}^{2}}{\left(1+\beta_{i} \beta_{e}\right)^{2}+\beta_{e}{ }^{2}}\right) f^{2}-f \phi_{1}^{\prime}\right) \\
& -\frac{\mathrm{DuKr}}{(1-\mathrm{DuScSr})} g_{1}-\frac{\operatorname{ReScDu} \cos \phi}{(1-\mathrm{DuScSr})} f g_{1}^{\prime}-2 \phi_{2}, \\
& \phi_{2}^{\prime \prime}=-\frac{\operatorname{Re} \operatorname{Pr} \cos \phi}{(1-\mathrm{DuScSr})}\left(\left(f^{\prime \prime}\right)^{2}\right. \\
& \left.+\left(\frac{\mathrm{Ha}^{2}}{\left(1+\beta_{i} \beta_{e}\right)^{2}+\beta_{e}{ }^{2}}\right)\left(f^{\prime}\right)^{2}+2 f^{\prime} \phi_{2}-f \phi_{2}^{\prime}\right) \\
& -\frac{\mathrm{DuKr}}{(1-\mathrm{DuScSr})} g_{2}-\frac{\operatorname{ReScDu} \cos \phi}{(1-\mathrm{DuScSr})}\left(f g_{2}^{\prime}\right. \\
& \left.-2 f^{\prime} g_{2}\right)
\end{aligned}
$$

$$
\begin{aligned}
& f \longrightarrow F_{1}[k], \\
& \phi_{1} \longrightarrow F_{2}[k], \\
& \phi_{2} \longrightarrow F_{3}[k], \\
& g_{1} \longrightarrow F_{4}[k],
\end{aligned}
$$

$$
\begin{aligned}
g_{1}^{\prime \prime} & =-2 g_{2}+\frac{\mathrm{Kr}}{(1-\mathrm{DuScSr})} g_{1}+\frac{\operatorname{ReScDu\operatorname {cos}\phi }}{(1-\mathrm{DuScSr})} f g_{1}^{\prime} \\
& +\frac{\operatorname{ReSc} \operatorname{Pr} \mathrm{Sr} \cos \phi}{(1-\mathrm{DuScSr})}\left(4\left(f^{\prime}\right)^{2}\right. \\
& \left.+\left(\frac{\mathrm{Ha}^{2}}{\left(1+\beta_{i} \beta_{e}\right)^{2}+\beta_{e}^{2}}\right) f^{2}-f \phi_{1}^{\prime}\right)
\end{aligned}
$$$$
g_{2}^{\prime \prime}=-\frac{\mathrm{Kr}}{(1-\mathrm{DuScSr})} g_{1}+\frac{\operatorname{ReSc} \operatorname{Pr} \mathrm{Sr} \cos \phi}{(1-\mathrm{DuScSr})}\left(\left(f^{\prime \prime}\right)^{2}\right.
$$$$
\left.+\left(\frac{\mathrm{Ha}^{2}}{\left(1+\beta_{i} \beta_{e}\right)^{2}+\beta_{e}^{2}}\right)\left(f^{\prime}\right)^{2}+2 f^{\prime} \phi_{2}-f \phi_{2}^{\prime}\right)
$$$$
+\frac{\operatorname{ReSc} \cos \phi}{(1-\mathrm{DuScSr})}\left(f g_{2}^{\prime}-2 f^{\prime} g_{2}\right)
$$

where prime denotes the differentiation with respect to $\lambda$ and the nondimensional boundary conditions in terms of $f, \phi_{1}$, $\phi_{2}, g_{1}$, and $g_{2}$ are

$$
\begin{aligned}
& f(0)=1-a, \\
& f^{\prime}(0)=0, \\
& g_{1}(0)=0, \\
& g_{2}(0)=0, \\
& \phi_{1}^{\prime}(0)=-\gamma \phi_{1}(0), \\
& \phi_{2}^{\prime}(0)=-\gamma \phi_{2}(0), \\
& f^{\prime}(1)=1, \\
& f^{\prime \prime}(1)=-\frac{f^{\prime}(1)}{\mathrm{Sl}}, \\
& \phi_{1}(1)=\frac{1}{\mathrm{Ec}}, \\
& \phi_{2}(1)=0, \\
& g_{1}(1)=\frac{1}{\mathrm{Sh}}, \\
& g_{2}(1)=0 .
\end{aligned}
$$

\section{Solution of the Problem}

The nonlinear equations (8) along with the boundary conditions (9) are transformed into recurrence equations (11)-(15) and initial values (16) using the transformations in (10) 

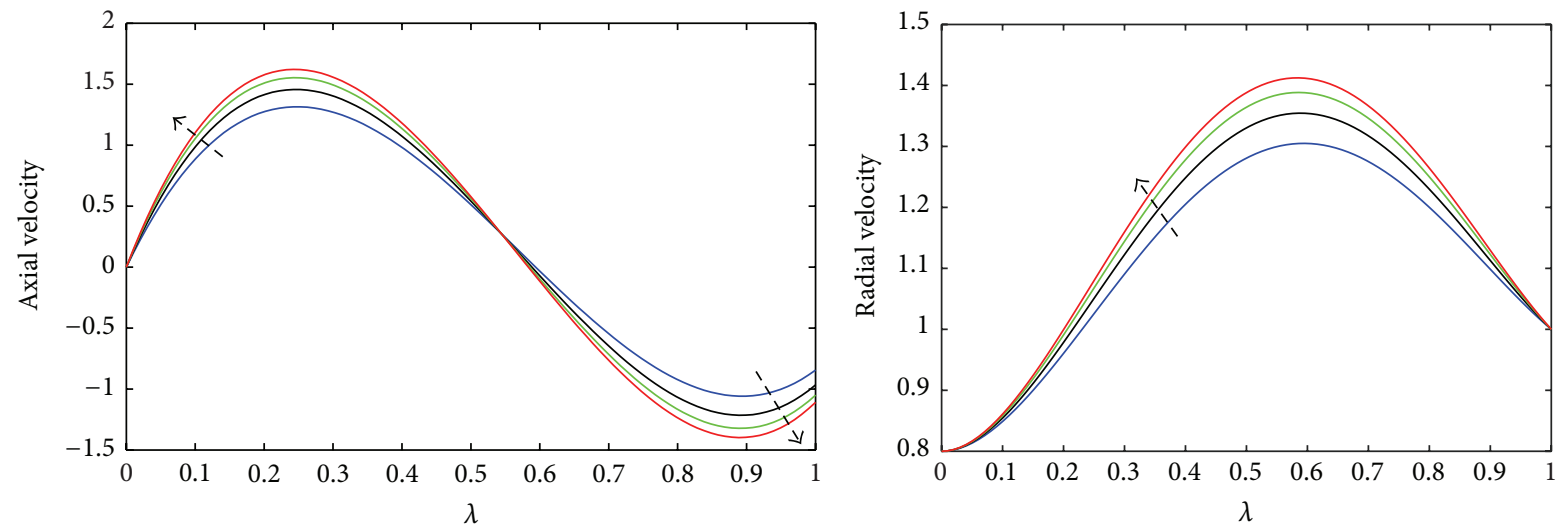

$-\mathrm{Wi}=1$

$W \mathrm{Wi}=3$

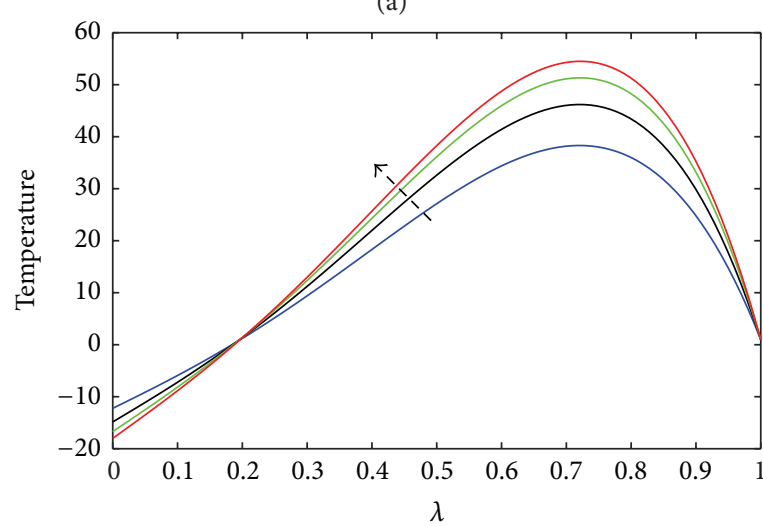

$\begin{aligned} \mathrm{Wi} & =1 \\ -\mathrm{Wi} & =3\end{aligned}$

Wi $=5$

$-\mathrm{Wi}=7$

(c)

- $\mathrm{Wi}=5$

$\mathrm{Wi}=7$

(a)
FIgURE 2: Effect of Wi on (a) axial velocity, (b) radial velocity, (c) temperature, and (d) concentration at $a=0.2 ; \mathrm{Re}=3 ; \phi=0.4 ; \mathrm{Ha}=4$; $\beta_{i}=8 ; \beta_{e}=0.2 ; \mathrm{Gr}=10 ; \mathrm{Gc}=10 ; \gamma=0.2 ; \mathrm{Du}=0.2 ; \mathrm{Sr}=0.2 ; \mathrm{Sc}=0.22 ; \mathrm{Kr}=0.2 ; \mathrm{Ec}=1 ; \mathrm{Sh}=1 ; \mathrm{Pr}=0.71 ; \mathrm{Sl}=0.2$.
$-\mathrm{Wi}=1$

$\mathrm{Wi}=3$

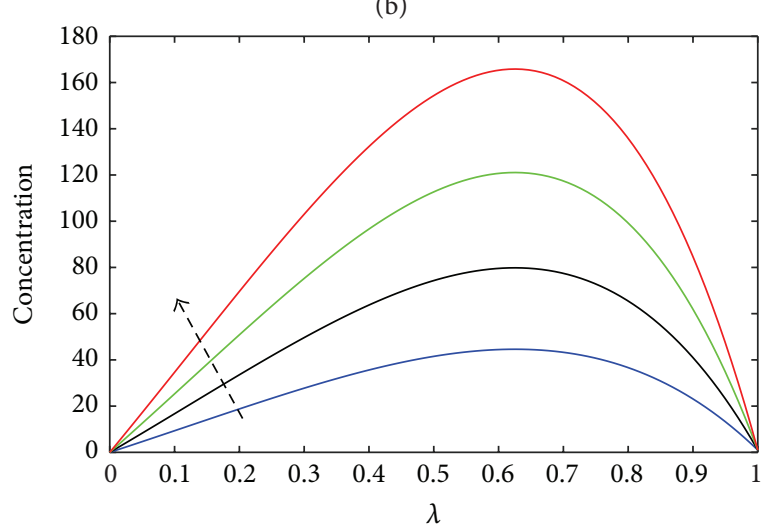

- $\mathrm{Wi}=1$

$-\mathrm{Wi}=5$

(d)

$$
\begin{aligned}
& g_{2} \longrightarrow F_{5}[k], \\
& f \phi_{1} \longrightarrow \sum_{l=0}^{k} F_{1}[l] F_{2}[k-l], \\
& f \phi_{1} \phi_{2} \longrightarrow \sum_{s=0}^{k} \sum_{m=0}^{k-s} F_{1}[s] F_{2}[m] F_{3}[k-s-m], \\
& F_{1}[k]=\frac{1}{k !} \frac{d^{k} f}{d x^{k}}, \quad f(x)=\sum_{j=0}^{k} F_{1}[j] x^{j}, \frac{d^{n} f}{d x^{n}} \longrightarrow \frac{(k+1)}{k !} F_{1}[k+n], \\
& F_{2}[k]=\frac{1}{k !} \frac{d^{k} \phi_{1}}{d x^{k}}, \quad \phi_{1}(x)=\sum_{j=0}^{k} F_{2}[j] x^{j}, \\
& F_{3}[k]=\frac{1}{k !} \frac{d^{k} \phi_{2}}{d x^{k}}, \quad \phi_{2}(x)=\sum_{j=0}^{k} F_{3}[j] x^{j}, \\
& F_{4}[k]=\frac{1}{k !} \frac{d^{k} g_{1}}{d x^{k}}, \quad g_{1}(x)=\sum_{j=0}^{k} F_{4}[j] x^{j}, \\
& F_{5}[k]=\frac{1}{k !} \frac{d^{k} g_{2}}{d x^{k}}, \quad g_{2}(x)=\sum_{j=0}^{k} F_{5}[j] x^{j},
\end{aligned}
$$




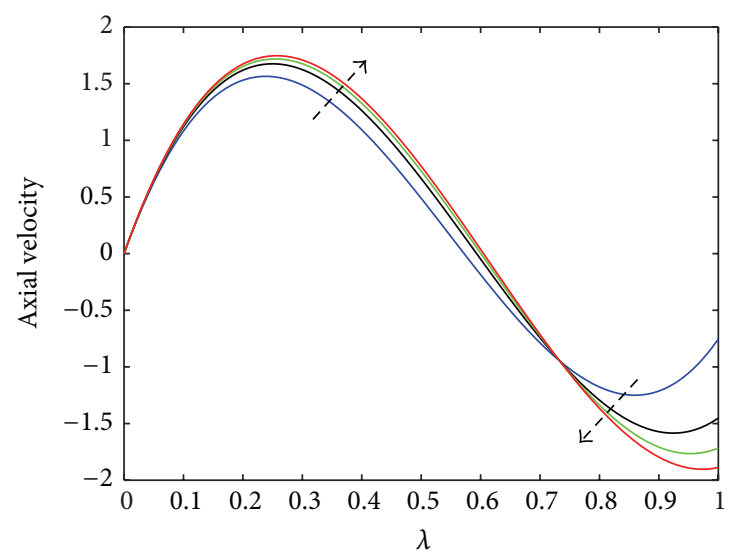

$-\mathrm{Sl}=0.1$

$-\mathrm{Sl}=0.4$

(a)

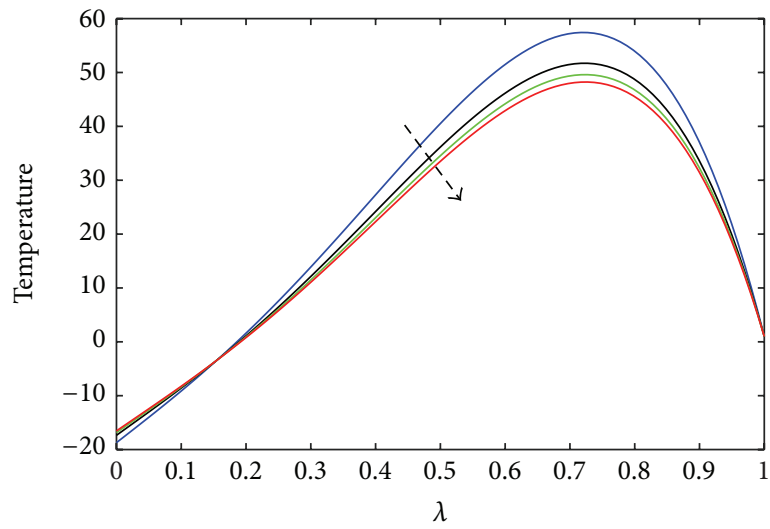

$\begin{aligned}-\mathrm{Sl} & =0.1 \\ -\mathrm{Sl} & =0.4\end{aligned}$

$-\mathrm{S} 1=0.8$

$-\mathrm{Sl}=1.6$

(c)

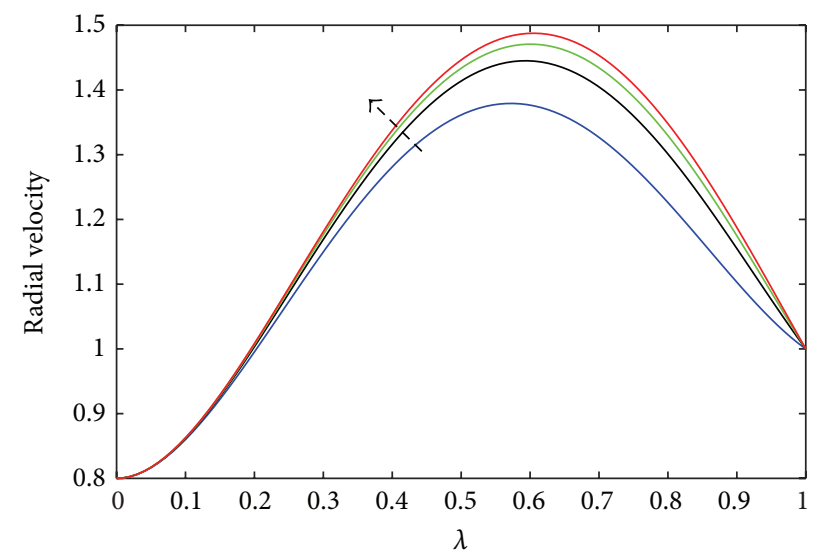

$-\mathrm{Sl}=0.1$

$-\mathrm{Sl}=0.8$

$-\mathrm{Sl}=1.6$

(b)

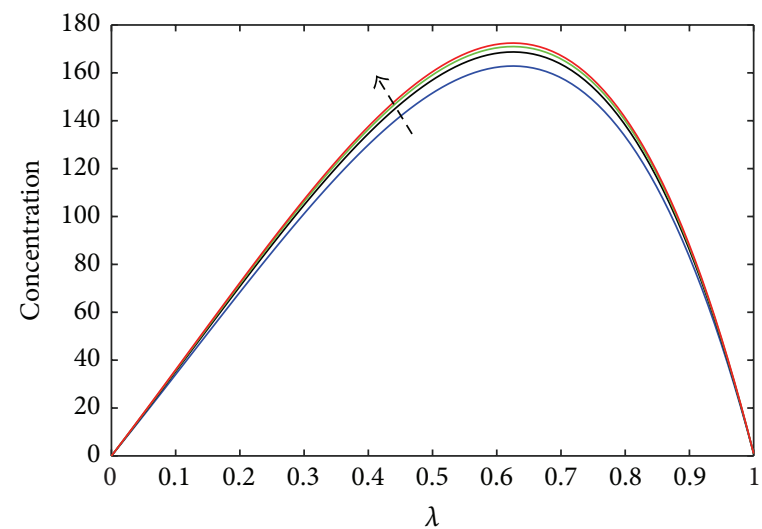

$\begin{aligned}-\mathrm{Sl} & =0.1 \\ -\mathrm{Sl} & =0.4\end{aligned}$

$-\mathrm{Sl}=0.8$

(d)

FIGURE 3: Effect of slip parameter on (a) axial velocity, (b) radial velocity, (c) temperature, and (d) concentration at $a=0.2 ; \mathrm{Re}=3$; Wi $=7$; $\phi=0.4 ; \mathrm{Ha}=4 ; \beta_{i}=8 ; \beta_{e}=0.2 ; \mathrm{Gr}=10 ; \mathrm{Gc}=10 ; \gamma=0.2 ; \mathrm{Du}=0.2 ; \mathrm{Sr}=0.2 ; \mathrm{Sc}=0.22 ; \mathrm{Kr}=0.2 ; \mathrm{Ec}=1 ; \mathrm{Sh}=1 ; \mathrm{Pr}=0.71$.

$F_{1}[k+4]=\left(\operatorname{Re} \cos \phi \sum_{l=0}^{k}\left((l+3)(l+2)(l+1) F_{1}[l+3] F_{1}[k-l]-(l+2)(l+1) F_{1}[l+2](k-l+1) F_{1}[k-l+1]\right)+\operatorname{Re} \operatorname{Wi} \cos 2 \phi\right.$

$\cdot \sum_{s=0}^{k-1} \sum_{m=0}^{k-s}(s+4)(s+3)(s+2)(s+1) F_{1}[m] F_{1}[s+4] F_{1}[k-m-s]-2 \operatorname{ReWi} \cos 2 \phi$

$\cdot \sum_{s=0}^{k} \sum_{m=0}^{k-s}\left((m+1)(s+1) F_{1}[m+1] F_{1}[s+1] F_{1}[k-m-s]+(m+2)(m+1)(s+2)(s+1) F_{1}[m+2] F_{1}[s+2] F_{1}[k-s-m]\right)$

$+\frac{\mathrm{Ha}^{2}\left(1+\beta_{i} \beta_{e}\right)}{\left(1+\beta_{i} \beta_{e}\right)^{2}+\beta_{e}^{2}}(k+2)(k+1) F_{1}[k+2]-\frac{\mathrm{Ec} \mathrm{Gr}}{\xi}(k+1)\left(F_{2}[k+1]+\xi^{2} F_{3}[k+1]\right)-\frac{\mathrm{Sh} \mathrm{Gc}}{\xi}(k+1)\left(F_{4}[k+1]\right.$

$\left.\left.+\xi^{2} F_{5}[k+1]\right)\right)\left(\left(1-F_{1}[0]^{2} \operatorname{ReWi} \cos 2 \phi\right)(k+4)(k+3)(k+2)(k+1)\right)^{-1}$, 


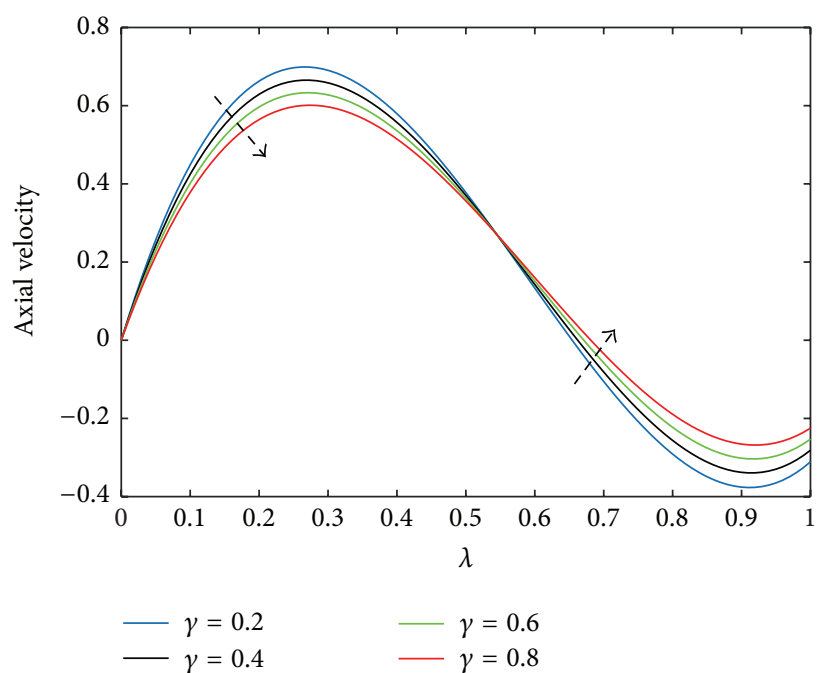

(a)

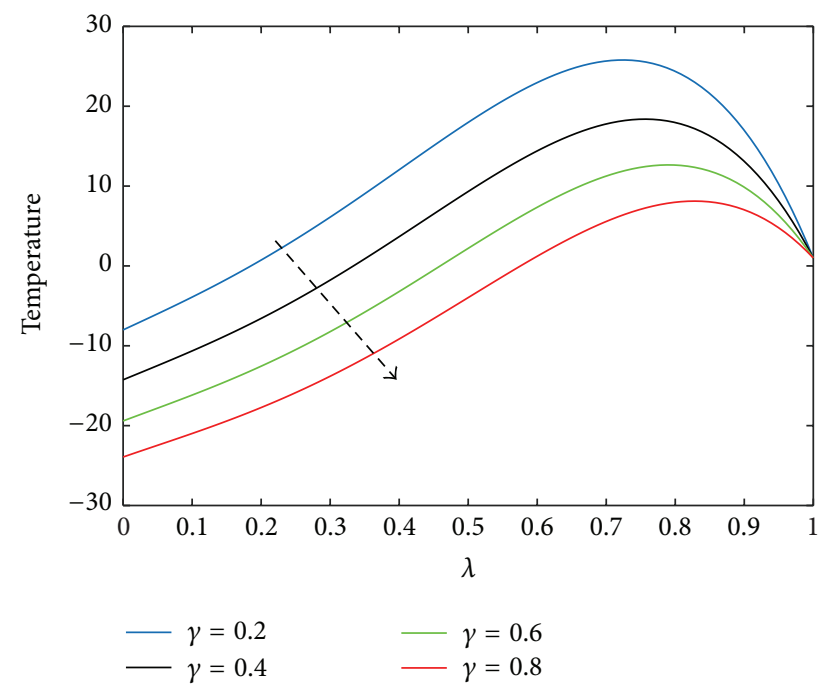

(c)

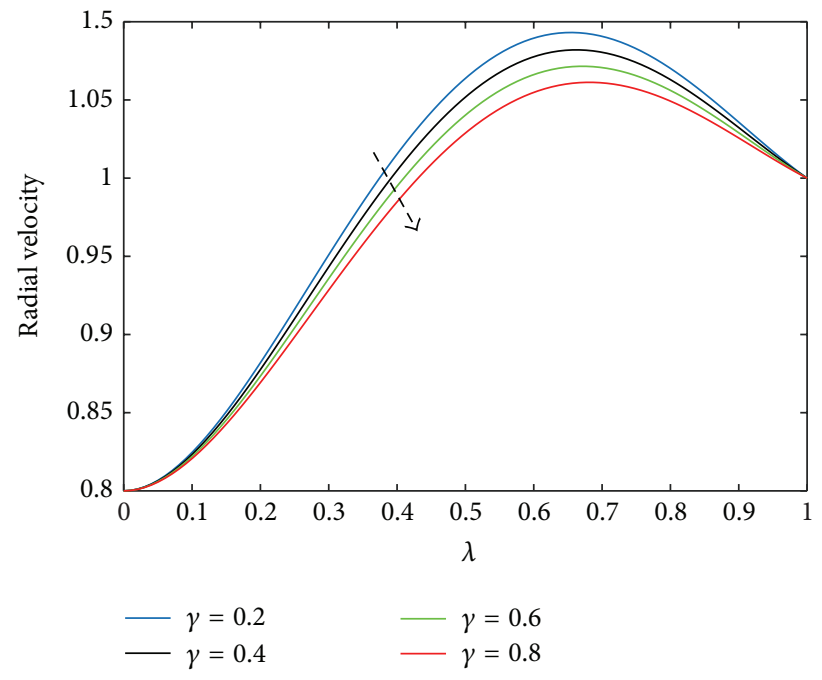

(b)
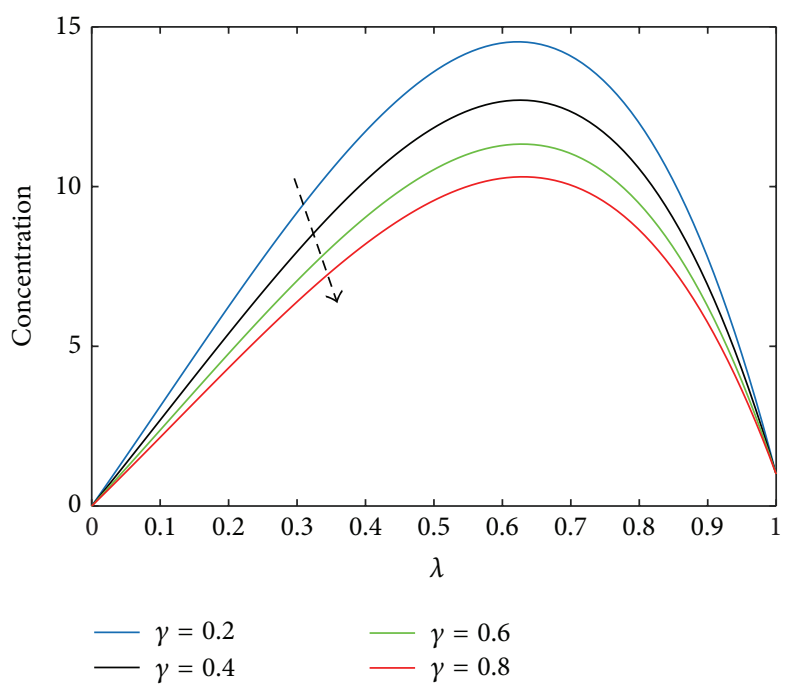

(d)

FIGURE 4: Effect of Biot number on (a) axial velocity, (b) radial velocity, (c) temperature, and (d) concentration at $a=0.2 ;$ Re $=3$; Wi $=1$; $\phi=0.4 ; \mathrm{Ha}=10 ; \beta_{i}=2 ; \beta_{e}=0.4 ; \mathrm{Gr}=10 ; \mathrm{Gc}=10 ; \mathrm{Du}=0.2 ; \mathrm{Sr}=0.2 ; \mathrm{Sc}=0.22 ; \mathrm{Kr}=0.2 ; \mathrm{Ec}=1 ; \mathrm{Sh}=1 ; \mathrm{Pr}=3 ; \mathrm{Sl}=0.2$.

$$
\begin{aligned}
& F_{2}[k+2]=\left(-\frac{\operatorname{Re} \operatorname{Pr} \cos \phi}{(1-\operatorname{DuScSr})} \sum_{l=0}^{k}\left(4(l+1) F_{1}[l+1](k-l+1) F_{1}[k-l+1]-(l+1) F_{2}[l+1] F_{1}[k-l]\right)-\frac{\operatorname{Re} \operatorname{Pr} \cos \phi}{(1-\mathrm{DuScSr})}\right. \\
& \left.\cdot \sum_{l=0}^{k}\left(\frac{\mathrm{Ha}^{2}}{\left(1+\beta_{i} \beta_{e}\right)^{2}+\beta_{e}^{2}}\right) F_{1}[l] F_{1}[k-l]-\frac{\mathrm{DuKr}}{(1-\mathrm{DuScSr})} F_{4}[k]-\frac{\operatorname{ReScDu} \cos \phi}{(1-\mathrm{DuScSr})} \sum_{l=0}^{k}(l+1) F_{4}[l+1] F_{1}[k-l]-2 F_{3}[k]\right)((k \\
& \text { +2) }(k+1))^{-1} \text {, } \\
& F_{3}[k+2]=\left(-\frac{\operatorname{Re} \operatorname{Pr} \cos \phi}{(1-\operatorname{DuScSr})}\left(\sum_{l=0}^{k}\left((l+2)(l+1) F_{1}[l+2](k-l+2)(k-l+1) F_{1}[k-l+2]\right)\right.\right. \\
& \left.+\sum_{l=0}^{k}\left(\frac{\mathrm{Ha}^{2}}{\left(1+\beta_{i} \beta_{e}\right)^{2}+\beta_{e}^{2}}\right)(l+1) F_{1}[l+1](k-l+1) F_{1}[k-l+1]+\sum_{l=0}^{k} 2(l+1) F_{1}[l+1] F_{3}[k-l]-(l+1) F_{3}[l+1] F_{1}[k-l]\right) \\
& \left.-\frac{\mathrm{DuKr}}{(1-\mathrm{DuScSr})} F_{5}[k]-\frac{\operatorname{ReScDu} \cos \phi}{(1-\mathrm{DuScSr})} \sum_{l=0}^{k}\left((l+1) F_{5}[l+1] F_{1}[k-l]-2(l+1) F_{1}[l+1] F_{5}[k-l]\right)\right)((k+2)(k+1))^{-1}
\end{aligned}
$$



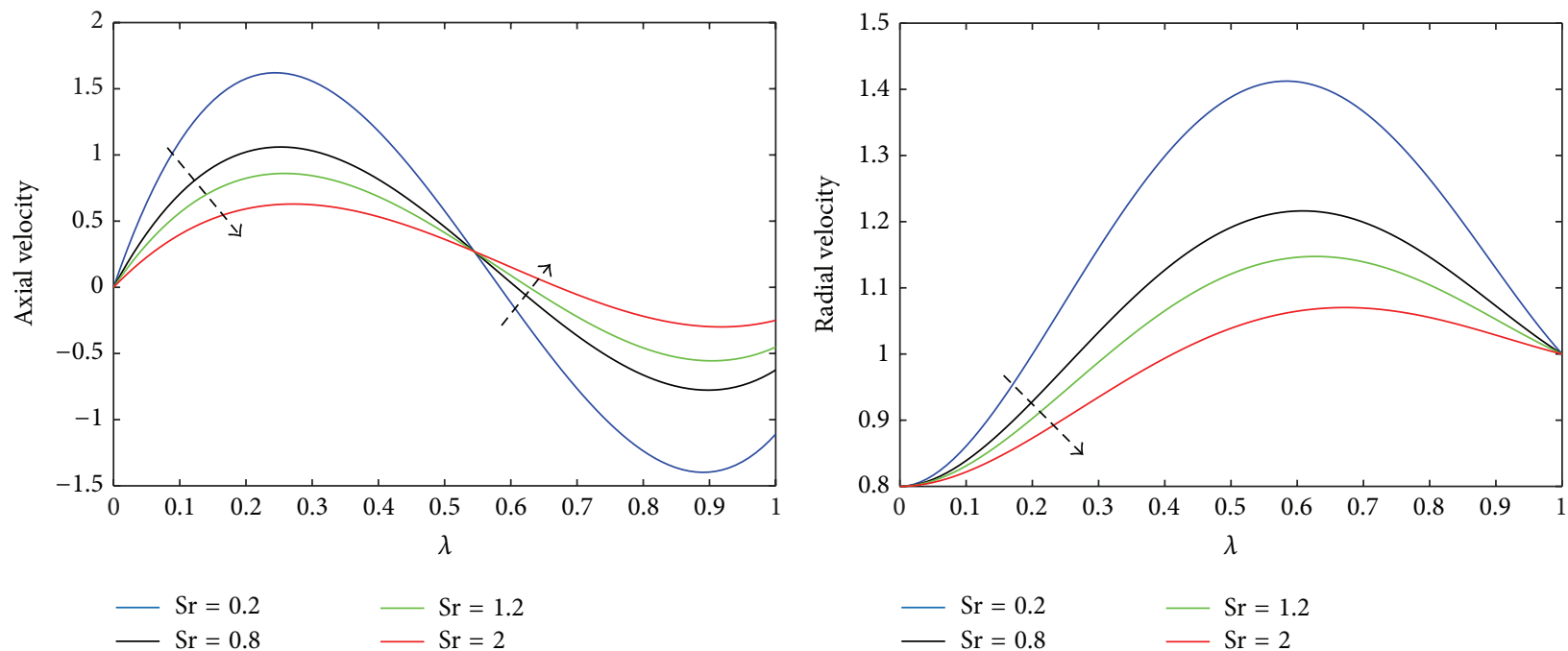

(a)

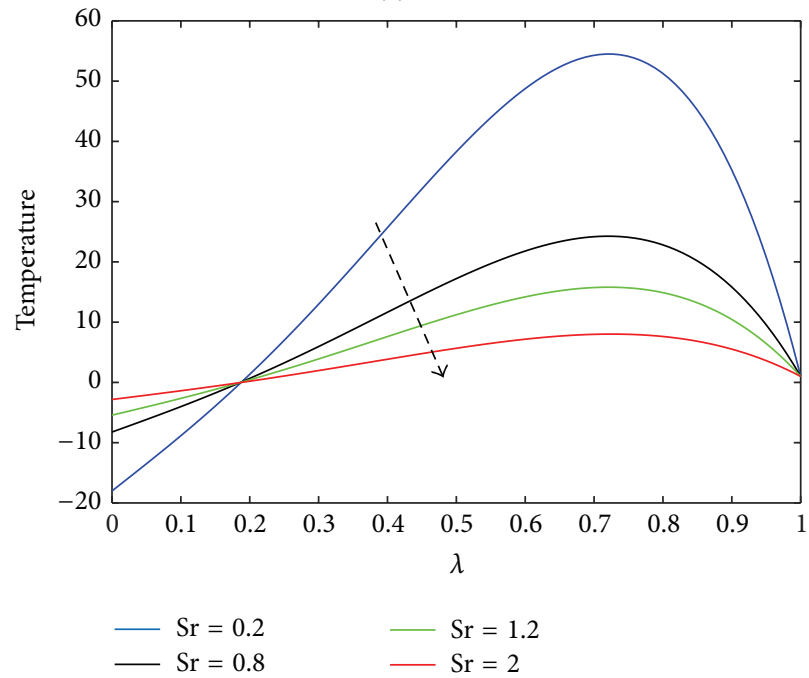

(c)

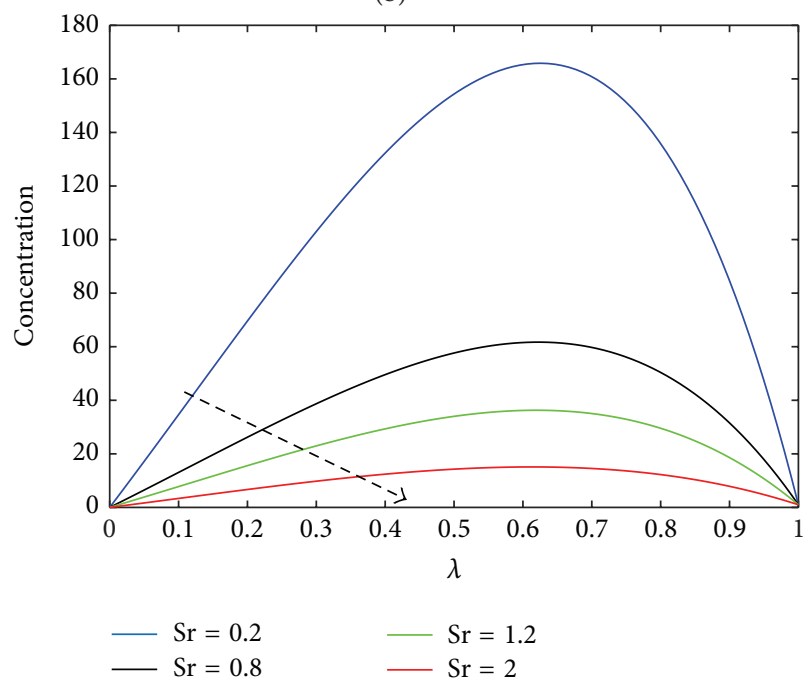

(d)

FIGURE 5: Effect of Sr on (a) axial velocity, (b) radial velocity, (c) temperature, and (d) concentration at $a=0.2 ; \mathrm{Re}=3$; Wi $=7 ; \phi=0.4$; $\mathrm{Ha}=4 ; \beta_{i}=8 ; \beta_{e}=0.2 ; \mathrm{Gr}=10 ; \mathrm{Gc}=10 ; \gamma=0.2 ; \mathrm{Du}=0.2 ; \mathrm{Sc}=0.22 ; \mathrm{Kr}=0.2 ; \mathrm{Ec}=1 ; \mathrm{Sh}=1 ; \mathrm{Pr}=0.71 ; \mathrm{Sl}=0.2$.

$$
\begin{aligned}
& F_{4}[k+2]=\left(-2 F_{5}[k]-\frac{\mathrm{Kr}}{(1-\mathrm{DuScSr})} F_{4}[k]-\frac{\operatorname{ReScDu} \cos \phi}{(1-\mathrm{DuScSr})} \sum_{l=0}^{k}(l+1) F_{4}[l+1] F_{1}[k-l]+\frac{\operatorname{ReSc} \operatorname{Pr} \operatorname{Sr} \cos \phi}{(1-\mathrm{DuScSr})}\right. \\
& \left.\cdot \sum_{l=0}^{k}\left(4(l+1) F_{1}[l+1](k-l+1) F_{1}[k-l+1]+\left(\frac{\mathrm{Ha}^{2}}{\left(1+\beta_{i} \beta_{e}\right)^{2}+\beta_{e}^{2}}\right) F_{1}[l] F_{1}[k-l]-(l+1) F_{2}[l+1] F_{1}[k-l]\right)\right)((k+2)(k \\
& +1))^{-1} \text {, } \\
& F_{5}[k+2]=\left(\frac{\operatorname{ReSc} \cos \phi}{(1-\mathrm{DuScSr})} \sum_{l=0}^{k}\left((l+1) F_{5}[l+1] F_{1}[k-l]-2(l+1) F_{1}[l+1] F_{5}[k-l]\right)+\frac{\operatorname{ReScDu} \cos \phi}{(1-\mathrm{DuScSr})}\right. \\
& \cdot \sum_{l=0}^{k}\left((l+2)(l+1) F_{1}[l+2](k-l+2)(k-l+1) F_{1}[k-l+2]\right)+\frac{\operatorname{ReSc} \operatorname{Pr} \operatorname{Srcos} \phi}{(1-\operatorname{DuScSr})} \\
& \cdot \sum_{l=0}^{k}\left(\left(\frac{\mathrm{Ha}^{2}}{\left(1+\beta_{i} \beta_{e}\right)^{2}+\beta_{e}{ }^{2}}\right)(l+1) F_{1}[l+1](k-l+1) F_{1}[k-l+1]\right)+\frac{\operatorname{ReSc} \operatorname{Pr} \operatorname{Sr} \cos \phi}{(1-\operatorname{DuScSr})} \sum_{l=0}^{k}\left(2(l+1) F_{1}[l+1] F_{3}[k-l]\right. \\
& \left.\left.-(l+1) F_{3}[l+1] F_{1}[k-l]\right)-\frac{\mathrm{Kr}}{(1-\mathrm{DuScSr})} F_{5}[k]\right)((k+2)(k+1))^{-1} \text {. }
\end{aligned}
$$



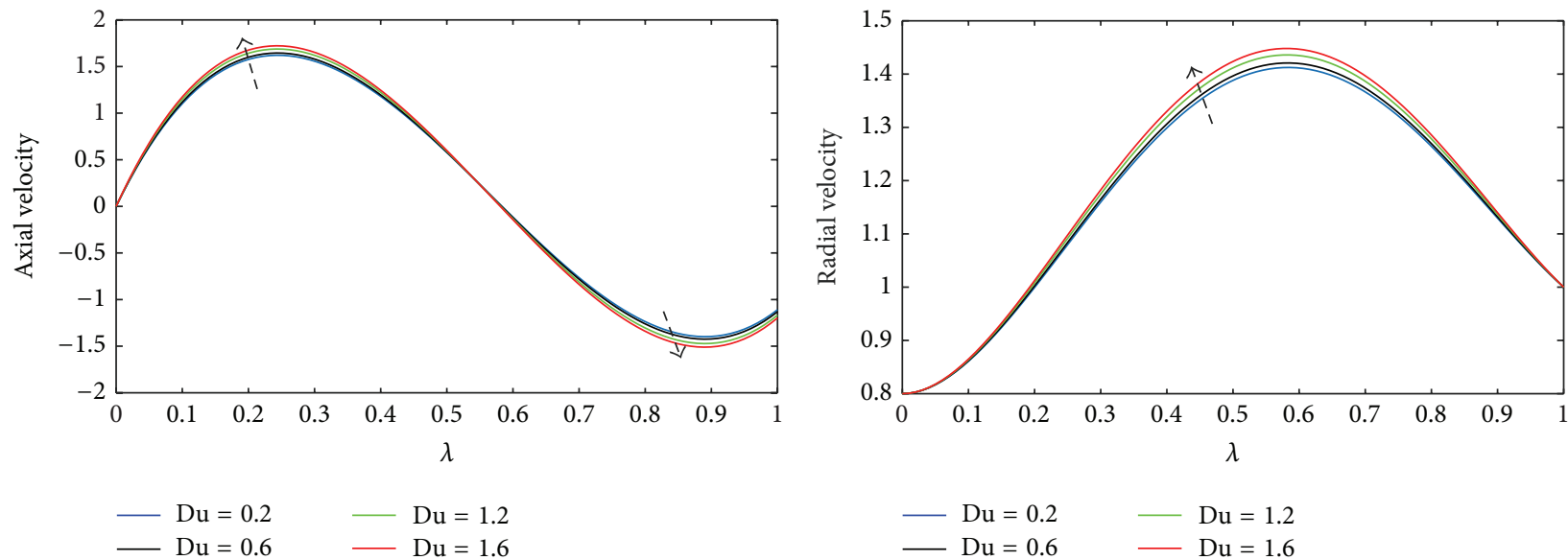

$\begin{aligned} & \mathrm{Du}=0.2-\mathrm{Du}=1.2 \\ & \mathrm{Du}=0.6 \quad-\mathrm{Du}=1.6\end{aligned}$

(a)

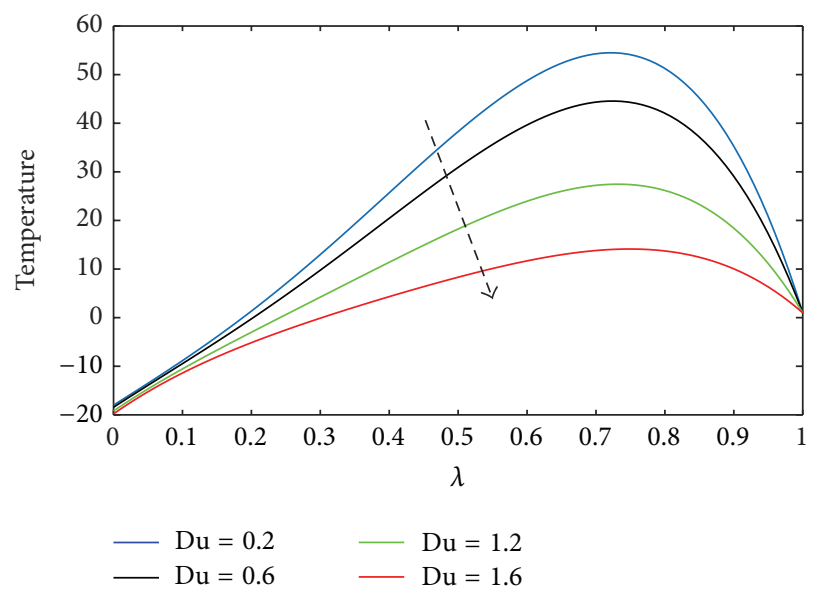

(c)

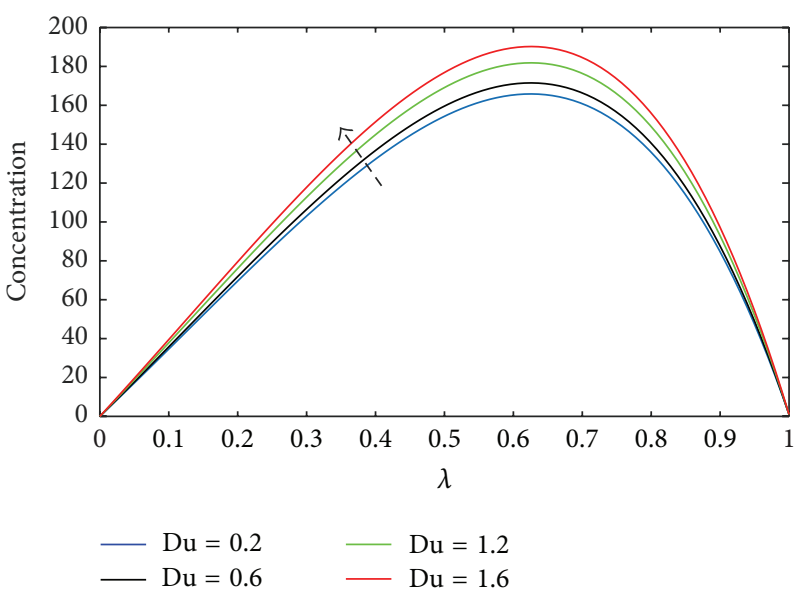

(d)

FIGURE 6: Effect of Du on (a) axial velocity, (b) radial velocity, (c) temperature, and (d) concentration at $a=0.2 ; \mathrm{Re}=3 ; \mathrm{Wi}=7 ; \phi=0.4$; $\mathrm{Ha}=4 ; \beta_{i}=8 ; \beta_{e}=0.2 ; \mathrm{Gr}=10 ; \mathrm{Gc}=10 ; \gamma=0.2 ; \mathrm{Sr}=0.2 ; \mathrm{Sc}=0.22 ; \mathrm{Kr}=0.2 ; \mathrm{Ec}=1 ; \mathrm{Sh}=1 ; \mathrm{Pr}=0.71 ; \mathrm{Sl}=0.2$.

The transformed equations of the boundary conditions are

$$
\begin{aligned}
& F_{1}[0]=1-a, \\
& F_{1}[1]=0, \\
& F_{2}[0]=n_{3}, \\
& F_{3}[0]=0, \\
& F_{4}[0]=0, \\
& F_{5}[0]=0, \\
& F_{1}[2]=n_{1}, \\
& F_{1}[3]=n_{2}, \\
& F_{2}[1]=\frac{\gamma}{\mathrm{Ec}}, \\
& F_{3}[1]=n_{4}, \\
& F_{4}[1]=n_{5}, \\
& F_{5}[0]=n_{6} .
\end{aligned}
$$

The values in (16) are used in the recurrence relations (11)(15) and $n_{1}, n_{2}, n_{3}, n_{4}, n_{5}$, and $n_{6}$ are calculated such that they satisfy the boundary conditions at $\lambda=1$; then we obtain nondimensional velocity components, temperature distribution, and concentration for various parameters by substituting these constants into the respective Taylor series.

\section{Results and Discussion}

In order to understand the flow characteristics in a better way, the numerical results for the nondimensional velocity components, temperature, and concentration with respect to various fluid and geometric parameters such as Weissenberg number Wi, slip parameter Sl, Biot number $\gamma$, Soret number Sr, Dufour number Du, Hall parameter $\beta_{e}$, ion slip parameter $\beta_{i}$, and Prandtl number Pr are calculated in the domain $[0,1]$ and presented in the form of graphs. A comparative study for the case of Newtonian fluid has been studied and the skin friction values using differential transform method and long series with polynomial coefficients as reported by Bujurke et al. [37] are presented in Table 1. 


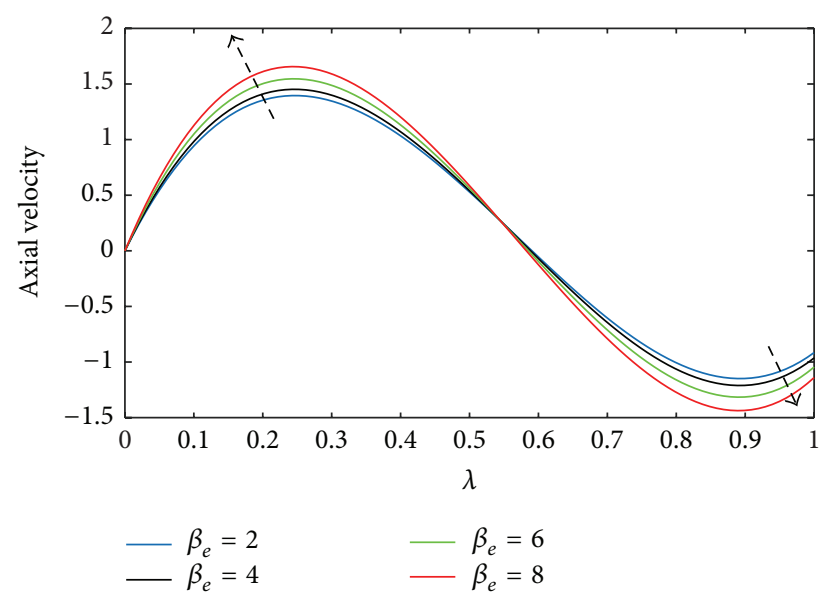

(a)

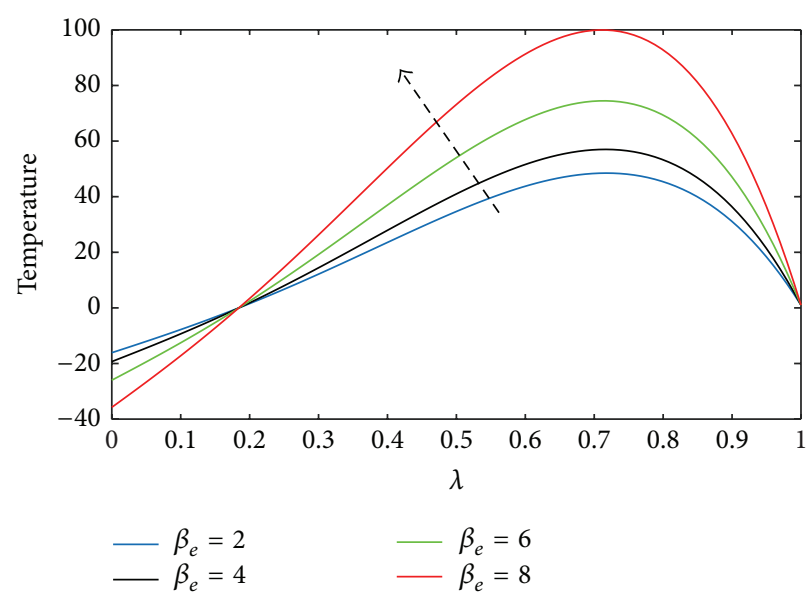

(c)

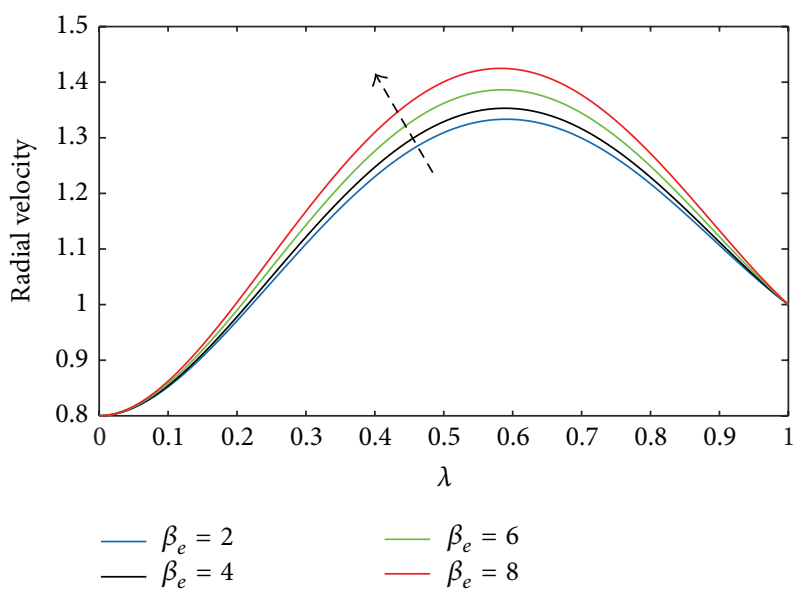

(b)

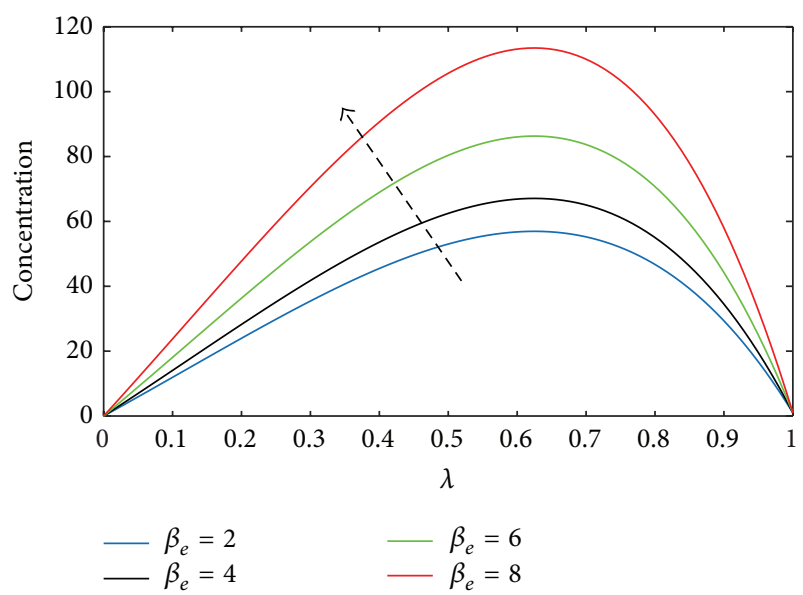

(d)

FIGURE 7: Effect of $\beta_{e}$ on (a) axial velocity, (b) radial velocity, (c) temperature, and (d) concentration at $a=0.2 ; \mathrm{Re}=3$; Wi $=1 ; \phi=0.4$; $\mathrm{Ha}=4 ; \beta_{i}=0.2 ; \mathrm{Gr}=10 ; \mathrm{Gc}=10 ; \gamma=0.2 ; \mathrm{Du}=0.2 ; \mathrm{Sr}=0.2 ; \mathrm{Sc}=0.22 ; \mathrm{Kr}=0.2 ; \mathrm{Ec}=1 ; \mathrm{Sh}=1 ; \mathrm{Pr}=0.71 ; \mathrm{Sl}=0.2$.

TABLE 1: Comparison of the present nondimensional skin friction values with Bujurke et al. [37] for Newtonian case.

\begin{tabular}{|c|c|c|c|c|}
\hline \multirow{2}{*}{ Serial number } & \multirow{2}{*}{$a$} & \multirow{2}{*}{$\mathrm{Re}$} & \multicolumn{2}{|l|}{$f^{\prime \prime}(0)$} \\
\hline & & & Bujurke et al. [37] & Present \\
\hline 1 & 0.04106 & 13.8833 & 0.0953 & 0.0954 \\
\hline 2 & 0.15420 & 31.3577 & 0.3190 & 0.3395 \\
\hline 3 & 0.00790 & 41.0960 & 0.0166 & 0.0176 \\
\hline 4 & 0.16480 & 52.9262 & 0.3300 & 0.3593 \\
\hline 5 & 0.01310 & 67.3621 & 0.0261 & 0.0298 \\
\hline
\end{tabular}

The effect of Weissenberg number Wi on velocity components, temperature distribution, and concentration is presented in Figure 2. It is observed that as Wi increases the radial velocity and concentration also increase towards the upper plate and the velocity in $X$-direction increases towards the center of the plates and then decreases. However, the temperature decreases near the lower plate and then increases towards the upper plate. It is a known fact that for low
Reynolds number flows $(0<\operatorname{Re}<5)$ the viscoelastic effect dominates the inertial effect.

Figure 3 gives the effect of slip parameter Sl on velocity components, temperature, and concentration. It is evident that as Sl increases, the velocity components and concentration are following the similar trend of Wi whereas the temperature is decreasing. It is due to the fact that the specific permeability decreases the velocity components of the fluid.

The effect of Biot number $\gamma$ on velocity components, temperature, and concentration is displayed in Figure 4. It is noticed that the radial velocity, temperature, and concentration profiles are decreasing with increasing $\gamma$ and the axial velocity is decreasing towards the center of the plates and then increases. Since $\gamma$ is inversely proportional to the thermal conductivity of the fluid, the heat transfer between the plates is decreased.

The effect of Soret number Sr on velocity components, temperature distribution, and concentration is studied in Figure 5. As Sr increases, the axial velocity decreases up to the center of the channel and then increases towards the 


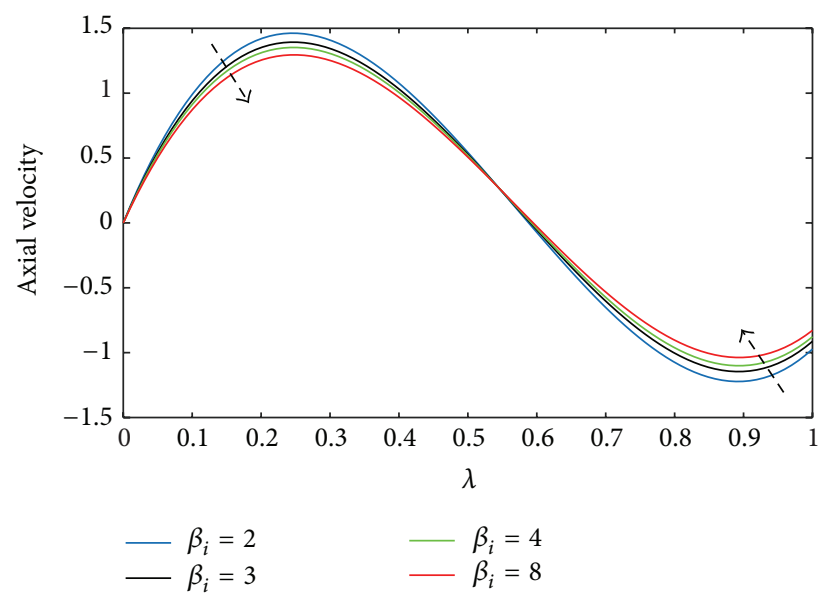

(a)

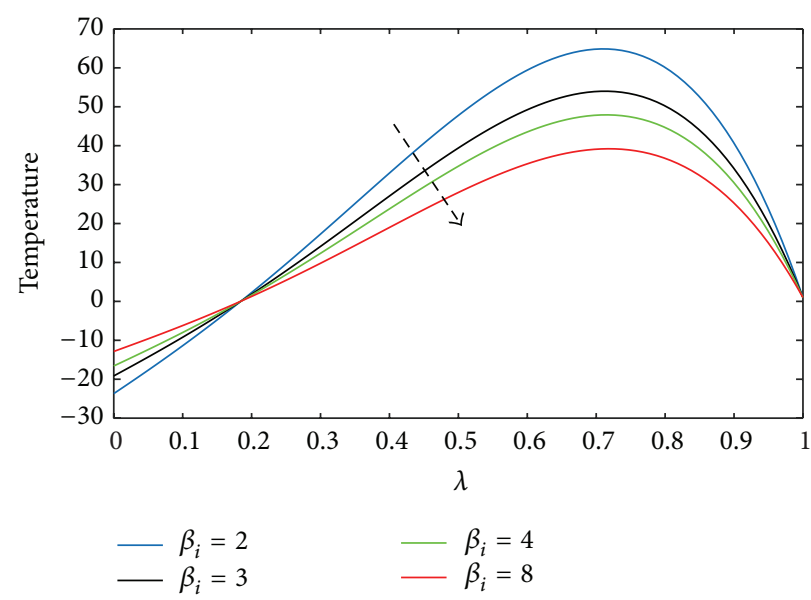

(c)

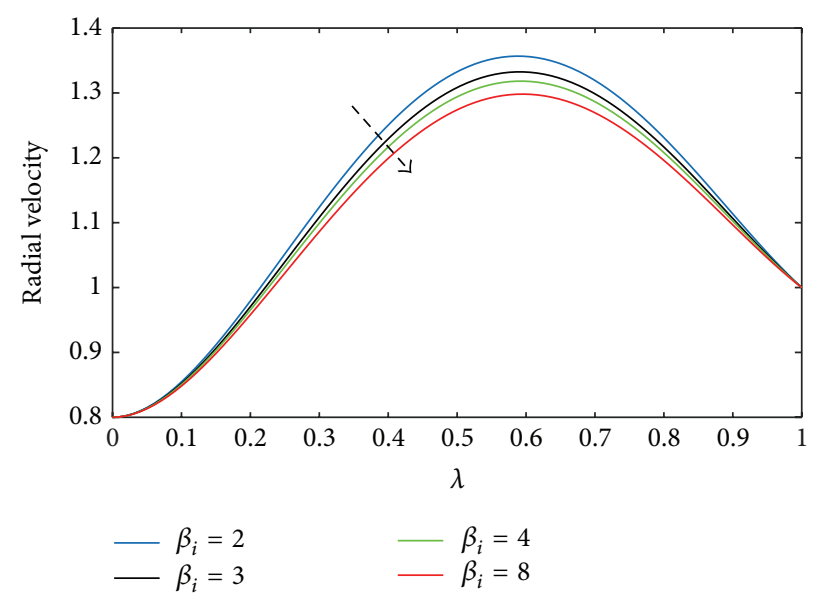

(b)

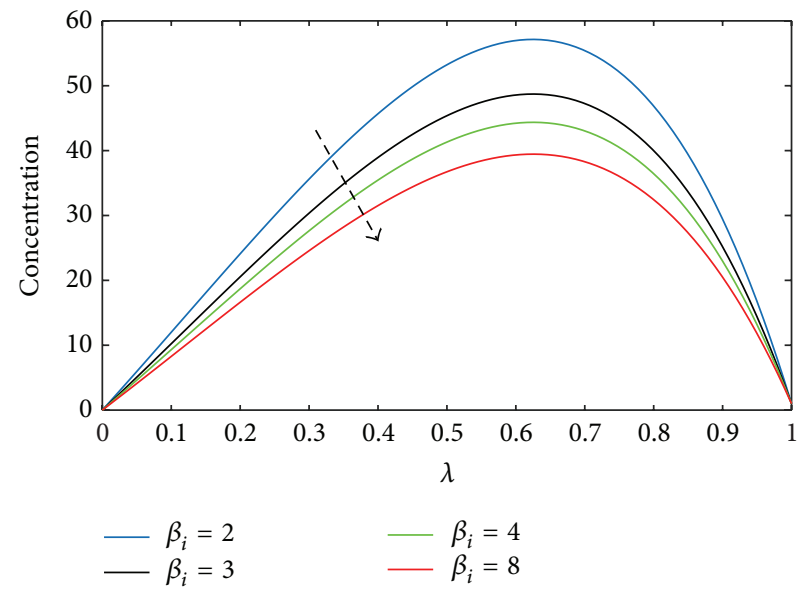

(d)

FIgure 8: Effect of $\beta_{i}$ on (a) axial velocity, (b) radial velocity, (c) temperature, and (d) concentration at $a=0.2 ; \mathrm{Re}=3$; Wi $=1 ; \phi=0.4$; $\mathrm{Ha}=10 ; \beta_{e}=0.4 ; \mathrm{Gr}=10 ; \mathrm{Gc}=10 ; \gamma=0.2 ; \mathrm{Du}=0.2 ; \mathrm{Sr}=0.2 ; \mathrm{Sc}=0.22 ; \mathrm{Kr}=0.2 ; \mathrm{Ec}=1 ; \mathrm{Sh}=1 ; \mathrm{Pr}=0.71 ; \mathrm{Sl}=0.2$.

upper plate, whereas the radial velocity and concentration of the fluid decrease towards the upper plate. However, the temperature distribution increases initially and then decreases. This is because of the fact that the mean heat transfer between the plates decreases with the increasing of thermal diffusion.

The effect of Dufour number Du on the velocity components, temperature, and concentration is shown in Figure 6. It is inferred that as Du increases, the radial velocity and concentration also increase whereas the temperature decreases. However the axial velocity increases towards the center of the channel and then decreases towards the upper plate. This is due to the fact that the concentration of the fluid is enhanced with diffusion in the temperature.

The effect of Hall parameter $\beta_{e}$ on velocity components, temperature distribution, and concentration is shown in Figure 7. $\beta_{e}$ increases the radial velocity and concentration also increases towards the upper plate, whereas the axial velocity increases towards the middle of the channel and then decreases. However, the temperature decreases near the lower plate and then increases towards the upper plate. This is because the effective conductivity decreases the damping force on the flow field.

Figure 8 represents the effect of ion slip parameter $\beta_{i}$ on velocity components, temperature, and concentration. It is observed that as $\beta_{i}$ increases, the velocity components, temperature, and concentration are following the opposite trend of $\beta_{e}$. This is because the increase in the ion slip scales down the effect of Lorentz force [38].

The effect of Prandtl number Pr on velocity components, temperature, and concentration is presented in Figure 9. It is analyzed that as $\mathrm{Pr}$ increases the axial velocity decreases towards the center of the plates and then increases, whereas the radial velocity and concentration are decreasing from the lower plate to the upper plate. However, the temperature increases near the lower plate and then decreases towards the upper plate. Physically, if $\mathrm{Pr}$ increases the thermal diffusivity decreases and this leads to the reduction in the heat transfer ability at the thermal boundary layer. 


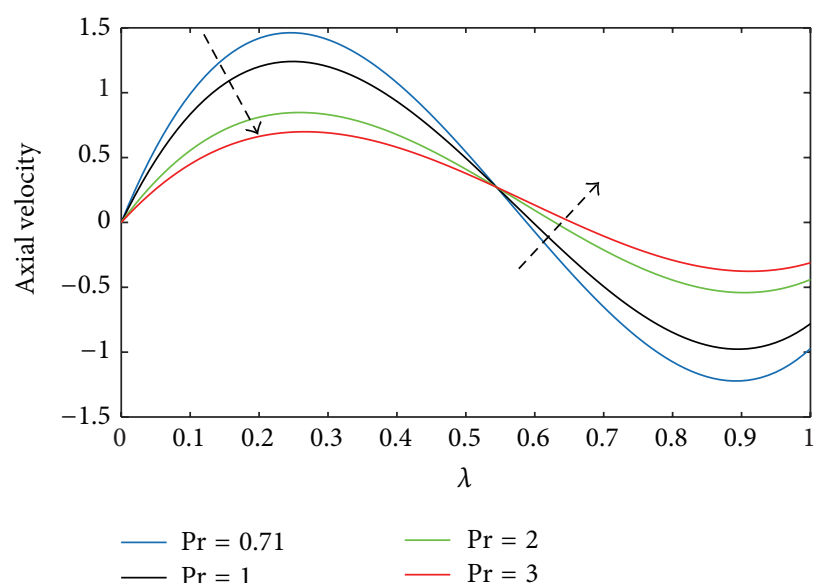

(a)

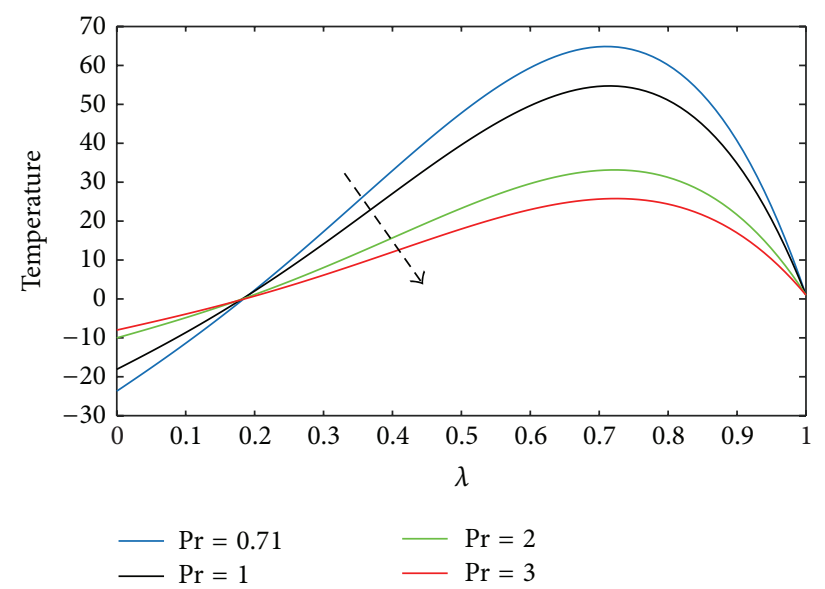

(c)

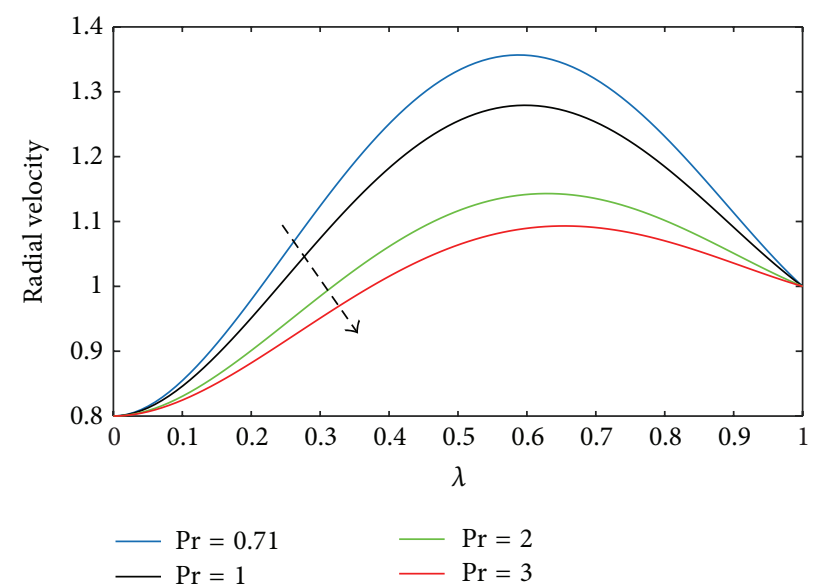

(b)

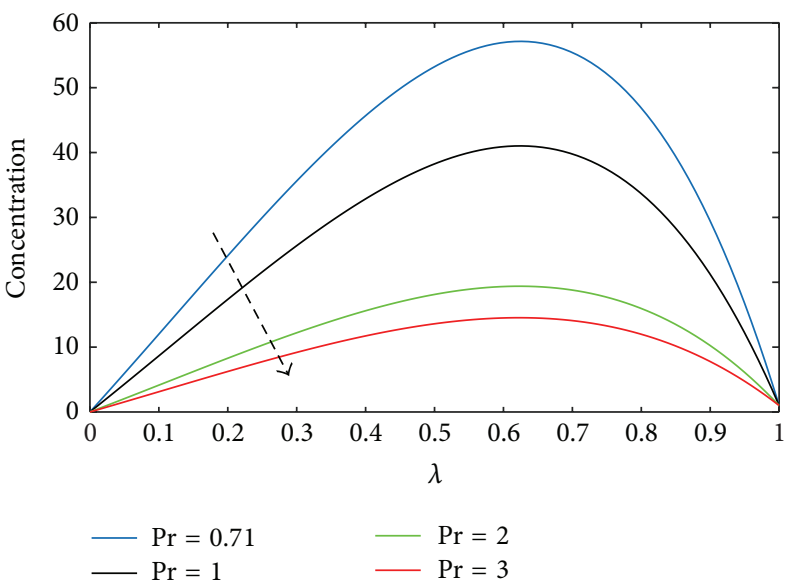

(d)

FIGURE 9: Effect of Pr on (a) axial velocity, (b) radial velocity, (c) temperature, and (d) concentration at $a=0.2 ; \mathrm{Re}=3$; Wi $=1 ; \phi=0.4$; $\mathrm{Ha}=10 ; \beta_{i}=2 ; \beta_{e}=0.4 ; \mathrm{Gr}=10 ; \mathrm{Gc}=10 ; \gamma=0.2 ; \mathrm{Du}=0.2 ; \mathrm{Sr}=0.2 ; \mathrm{Sc}=0.22 ; \mathrm{Kr}=0.2 ; \mathrm{Ec}=1 ; \mathrm{Sh}=1 ; \mathrm{Sl}=0.2$.

\section{Conclusions}

The effects of thermal-diffusion and diffusion-thermo on an unsteady free convective chemically reacting upper convected Maxwell fluid flow between two porous parallel plates with Hall and ion slip currents under the influence of slip and convective boundary conditions are considered. The flow field equations are reduced to nonlinear ordinary differential equations by using similarity transformations and then solved by differential transform method. The results are obtained for the nondimensional velocity components, temperature, and concentration distributions with respect to various fluid and geometric parameters such as $\mathrm{Wi}, \mathrm{Sl}, \gamma, \mathrm{Sr}$, $\mathrm{Du}, \beta_{e}, \beta_{i}$, and $\operatorname{Pr}$ and we observe the following:

(i) The concentration of the fluid is enhanced with slip parameter, whereas the temperature of the fluid is decreased with increasing of Biot number.

(ii) Soret and Dufour parameters exhibit opposite trend on concentration distribution.

(iii) The Weissenberg number and Hall parameter show similar effects on velocity components. (iv) The Hall and ion slip parameters exhibit reverse trend on temperature and concentration distributions.

(v) The present results are compared with the previously published work [37] and are found to be in good agreement.

These results have possible applications in science and technology such as fermentation, biorheology, geophysics, polymer industry, composite processing, food processing, and petroleum industries.

\section{Nomenclature}

$h$ : Distance between parallel plates

$V_{1} e^{i \omega t}:$ Injection velocity at lower plate

$V_{2} e^{i \omega t}$ : Suction velocity at the upper plate

$a: \quad$ Suction injection parameter, $1-V_{2} / V_{1}$

$P: \quad$ Fluid pressure

$u(x, y)$ : Axial velocity component

$v(x, y)$ : Velocity component in $y$-direction

Wi: Weissenberg number, $\beta V_{2} / h$ 


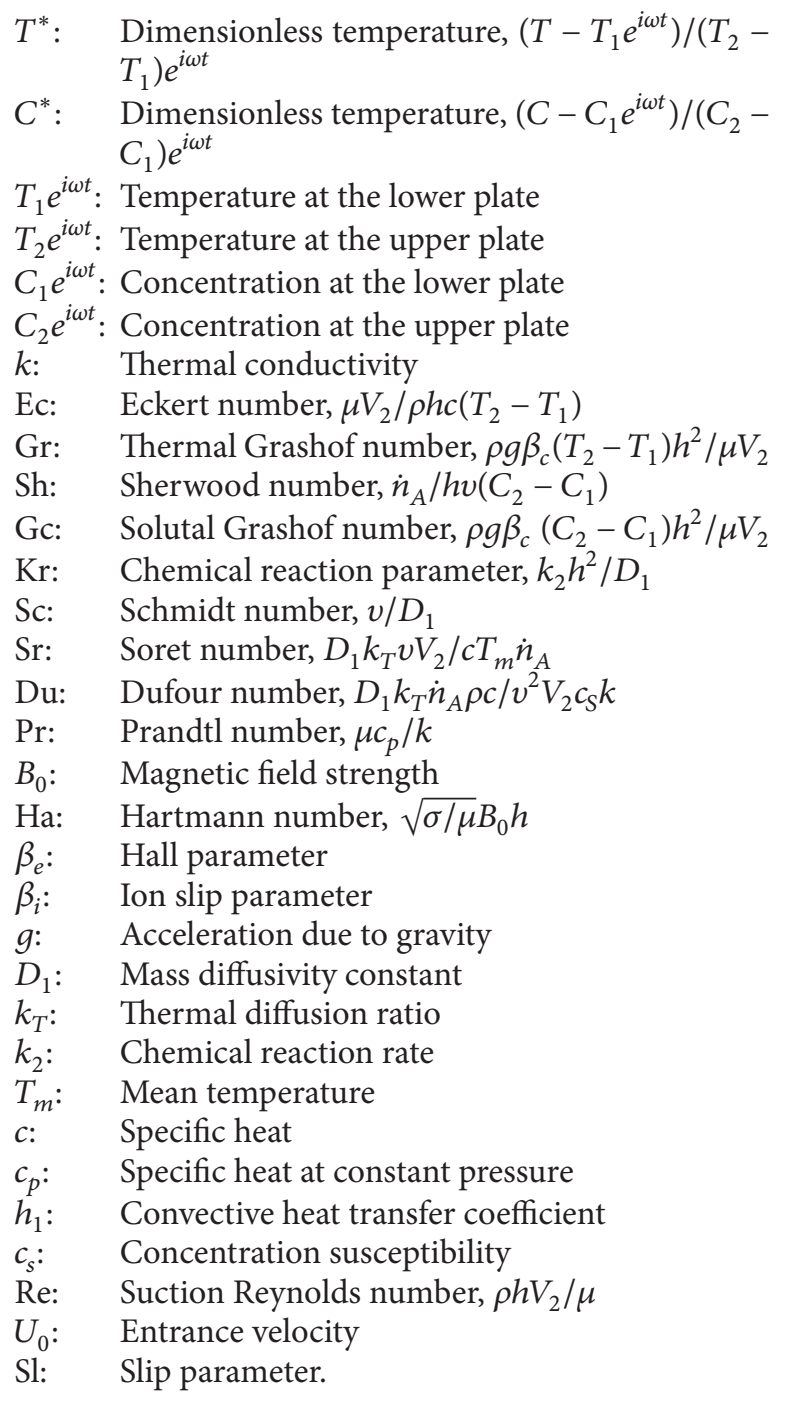

\section{Greek Letters}

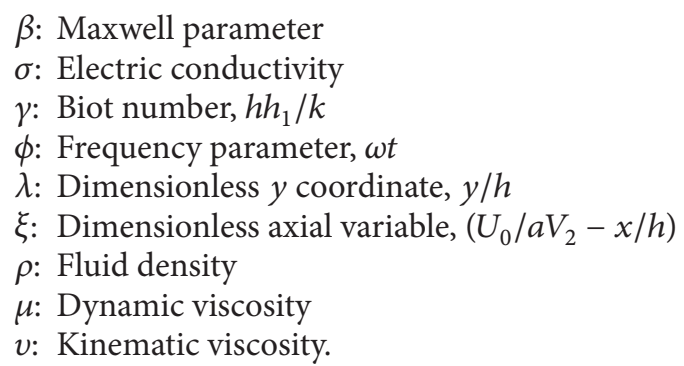

\section{Competing Interests}

The authors declare that there is no conflict of interests regarding the publication of this paper.

\section{Acknowledgments}

One of the authors (Pravin Kashyap Kambhatla) is grateful to the University Grants Commission, Government of India, for providing financial support in the form of Senior Research Fellowship (F.2-18/2012(SA-I)).

\section{References}

[1] A. S. Berman, "Laminar flow in channels with porous walls," Journal of Applied Physics, vol. 24, pp. 1232-1235, 1953.

[2] F. M. White Jr., B. F. Barfield, and M. J. Goglia, "Laminar flow in a uniformly porous channel," Journal of Applied Mechanics, vol. 25, no. 4, pp. 613-617, 1958.

[3] G. Walker and T. Davies, "Mass transfer in laminar flow between parallel permeable plates," AIChE Journal, vol. 20, no. 5, pp. 881889,1974

[4] E. A. Hamza, "Suction and injection effects on a similar flow between parallel plates," Journal of Physics D: Applied Physics, vol. 32, no. 6, pp. 656-663, 1999.

[5] D. Srinivasacharya, J. V. Ramana Murthy, and D. Venugopalam, "Unsteady stokes flow of micropolar fluid between two parallel porous plates," International Journal of Engineering Science, vol. 39, no. 14, pp. 1557-1563, 2001.

[6] M. Sheikholeslami, M. Hatami, and D. D. Ganji, "Micropolar fluid flow and heat transfer in a permeable channel using analytical method," Journal of Molecular Liquids, vol. 194, pp. 30-36, 2014.

[7] Y.-L. Chen and K.-Q. Zhu, "Couette-Poiseuille flow of Bingham fluids between two porous parallel plates with slip conditions," Journal of Non-Newtonian Fluid Mechanics, vol. 153, no. 1, pp. 1-11, 2008.

[8] K. D. Singh and R. Pathak, "Effect of slip conditions and Hall current on an oscillatory convective MHD flow in a rotating vertical porous channel with thermal radiation," International Journal of Applied Mathematics and Mechanics, vol. 9, pp. 6077, 2013.

[9] A. N. Bhat and M. L. Mittal, "Heat transfer in a MHD channel with uniform wall heat flux-effects of Hall and ion slip currents," International Journal of Heat and Mass Transfer, vol. 23, no. 7, pp. 919-926, 1980.

[10] D. Srinivasacharya and K. Kaladhar, "Mixed convection flow of couple stress fluid between parallel vertical plates with Hall and Ion-slip effects," Communications in Nonlinear Science and Numerical Simulation, vol. 17, no. 6, pp. 2447-2462, 2012.

[11] A. K. Singh and R. S. R. Gorla, "Free convection heat and mass transfer with Hall current, Joule heating and thermal diffusion," Heat and Mass Transfer, vol. 45, no. 11, pp. 1341-1349, 2009.

[12] A. Raptis, C. Massalas, and G. Tzivanidis, "Hydromagnetic free convection flow through a porous medium between two parallel plates," Physics Letters A, vol. 90, no. 6, pp. 288-289, 1982.

[13] O. Abdulaziz and I. Hashim, "Fully developed free convection heat and mass transfer of a micropolar fluid between porous vertical plates," Numerical Heat Transfer; Part A: Applications, vol. 55, no. 3, pp. 270-288, 2009.

[14] O. D. Makinde and A. Aziz, "MHD mixed convection from a vertical plate embedded in a porous medium with a convective boundary condition," International Journal of Thermal Sciences, vol. 49, no. 9, pp. 1813-1820, 2010.

[15] S. Yao, T. Fang, and Y. Zhong, "Heat transfer of a generalized stretching/shrinking wall problem with convective boundary conditions," Communications in Nonlinear Science and Numerical Simulation, vol. 16, no. 2, pp. 752-760, 2011.

[16] C. RamReddy, T. Pradeepa, and D. Srinivasacharya, "Similarity solution for free convection flow of a micropolar fluid under convective boundary condition via Lie scaling group transformations," Advances in High Energy Physics, vol. 2015, Article ID 650813, 16 pages, 2015. 
[17] A. Postelnicu, "Heat and mass transfer by natural convection at a stagnation point in a porous medium considering Soret and Dufour effects," Heat and Mass Transfer, vol. 46, no. 8-9, pp. 831840, 2010.

[18] T. Hayat, T. Muhammad, S. A. Shehzad, and A. Alsaedi, "Soret and Dufour effects in three-dimensional flow over an exponentially stretching surface with porous medium, chemical reaction and heat source/sink," International Journal of Numerical Methods for Heat and Fluid Flow, vol. 25, no. 4, pp. 762-781, 2015.

[19] A. J. Chamkha and A. M. Rashad, "Unsteady heat and mass transfer by MHD mixed convection flow from a rotating vertical cone with chemical reaction and Soret and Dufour effects," The Canadian Journal of Chemical Engineering, vol. 92, no. 4, pp. 758-767, 2014.

[20] O. Ojjela and N. Naresh Kumar, "Unsteady MHD mixed convective flow of chemically reacting and radiating couple stress fluid in a porous medium between parallel plates with Soret and Dufour effects," Arabian Journal for Science and Engineering, vol. 41, no. 5, pp. 1941-1953, 2016.

[21] J. G. Oldroyd, "On the formulation of rheological equations of state," Proceedings of the Royal Society of London, Series A: Mathematical, Physical and Engineering Sciences, vol. 200, pp. 523-591, 1950.

[22] M. Awais, T. Hayat, A. Alsaedi, and S. Asghar, "Time-dependent three-dimensional boundary layer flow of a Maxwell fluid," Computers and Fluids, vol. 91, pp. 21-27, 2014.

[23] J. J. Choi, Z. Rusak, and J. A. Tichy, "Maxwell fluid suction flow in a channel," Journal of Non-Newtonian Fluid Mechanics, vol. 85 , no. 2-3, pp. 165-187, 1999.

[24] T. Hayat, N. Ali, and S. Asghar, "Hall effects on peristaltic flow of a Maxwell fluid in a porous medium," Physics Letters A, vol. 363, no. 5-6, pp. 397-403, 2007.

[25] Z. Abbas, M. Sajid, and T. Hayat, "MHD boundary-layer flow of an upper-convected Maxwell fluid in a porous channel," Theoretical and Computational Fluid Dynamics, vol. 20, no. 4, pp. 229-238, 2006.

[26] S. Mukhopadhyay and R. S. Gorla, "Unsteady MHD boundary layer flow of an upper convected Maxwell fluid past a stretching sheet with first order constructive/destructive chemical reaction," Journal of Naval Architecture and Marine Engineering, vol. 9, no. 2, pp. 123-133, 2012.

[27] T. Hayat and Z. Abbas, "Channel flow of a Maxwell fluid with chemical reaction," Zeitschrift für ange-wandte Mathematik und Physik, vol. 59, no. 1, pp. 124-144, 2008.

[28] A. Alizadeh-Pahlavan and K. Sadeghy, "On the use of homotopy analysis method for solving unsteady MHD flow of Maxwellian fluids above impulsively stretching sheets," Communications in Nonlinear Science and Numerical Simulation, vol. 14, no. 4, pp. 1355-1365, 2009.

[29] J. K. Zhou, Differential Transformation and Its Applications for Electrical Circuits, Huazhong University Press, Wuhan, China, 1986.

[30] C. K. Chen and S. H. Ho, "Solving partial differential equations by two-dimensional differential transform method," Applied Mathematics and Computation, vol. 106, no. 2-3, pp. 171-179, 1999.

[31] F. Ayaz, "Solutions of the system of differential equations by differential transform method," Applied Mathematics and Computation, vol. 147, no. 2, pp. 547-567, 2004.

[32] S. Mosayebidorcheh, M. Vatani, D. D. Ganji, and T. Mosayebidorcheh, "Investigation of the viscoelastic flow and species diffusion in a porous channel with high permeability," Alexandria Engineering Journal, vol. 53, no. 4, pp. 779-785, 2014.

[33] M. Sheikholeslami, M. M. Rashidi, D. M. Al Saad, F. Firouzi, H. B. Rokni, and G. Domairry, "Steady nanofluid flow between parallel plates considering thermophoresis and Brownian effects," Journal of King Saud University -Science, 2015.

[34] M. Sheikholeslami and D. D. Ganji, "Nanofluid flow and heat transfer between parallel plates considering Brownian motion using DTM," Computer Methods in Applied Mechanics and Engineering, vol. 283, pp. 651-663, 2015.

[35] M. Hatami and D. Jing, "Differential transformation method for newtonian and non-newtonian nanofluids flow analysis: compared to numerical solution," Alexandria Engineering Journal, 2016.

[36] S. H. Esmail and M. M. Taha, "Application of the differential transform method to the advection-diffusion equation in threedimensions," Progress in Physics, vol. 12, no. 3, pp. 205-210, 2016.

[37] N. M. Bujurke, V. S. Madalli, and B. G. Mulimani, "Long series analysis of laminar flow through parallel and uniformly porous walls of different permeability," Computer Methods in Applied Mechanics and Engineering, vol. 160, no. 1-2, pp. 39-56, 1998.

[38] G. W. Sutton and A. Sherman, Engineering Magnetohydrodynamics, McGraw-Hill, New York, NY, USA, 1965. 


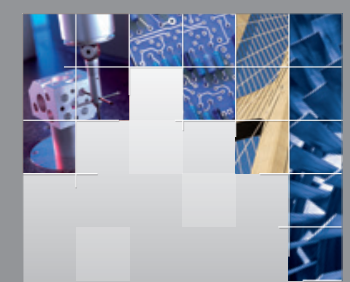

\section{Enfincering}
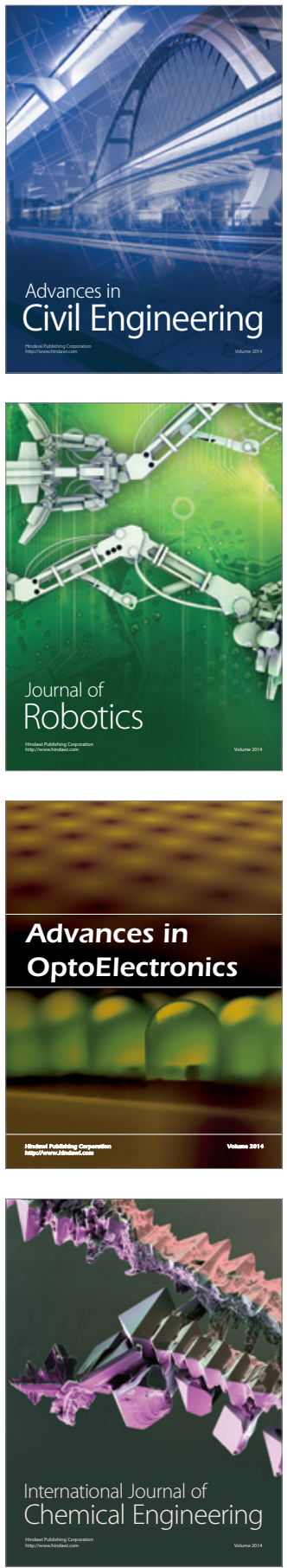

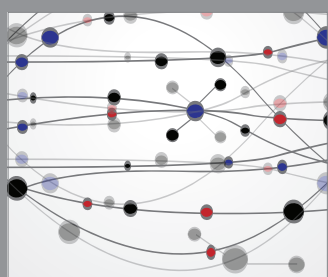

The Scientific World Journal

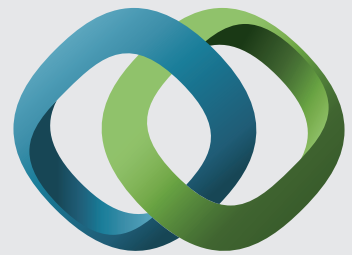

\section{Hindawi}

Submit your manuscripts at

http://www.hindawi.com
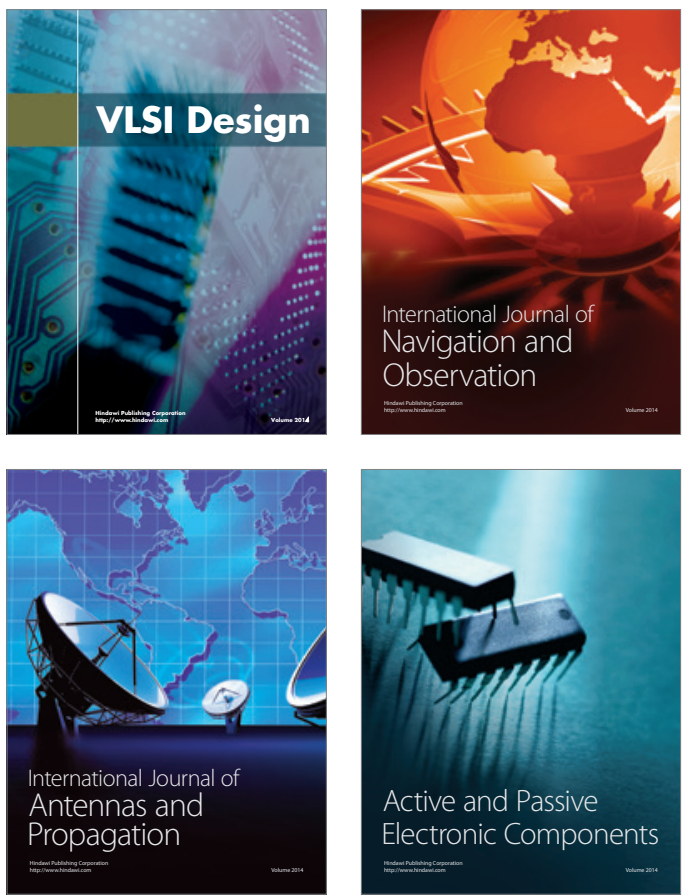
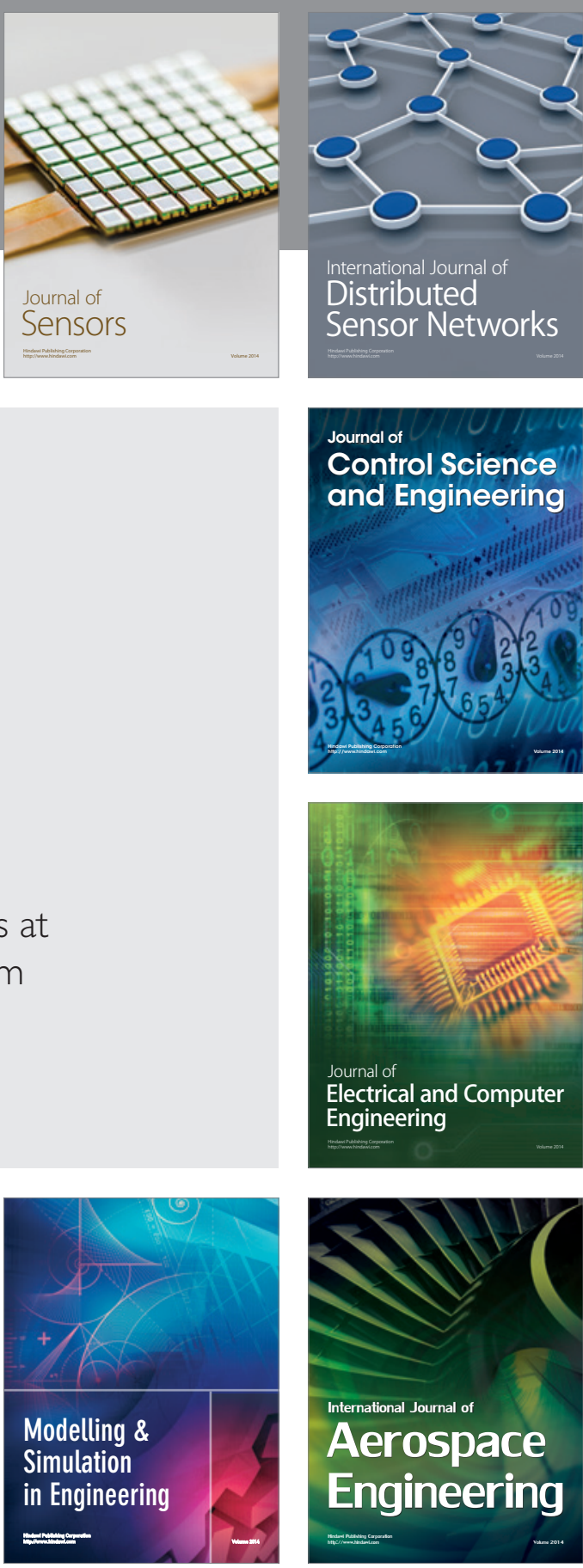

International Journal of

Distributed

Sensor Networks

Journal of

Control Science

and Engineering
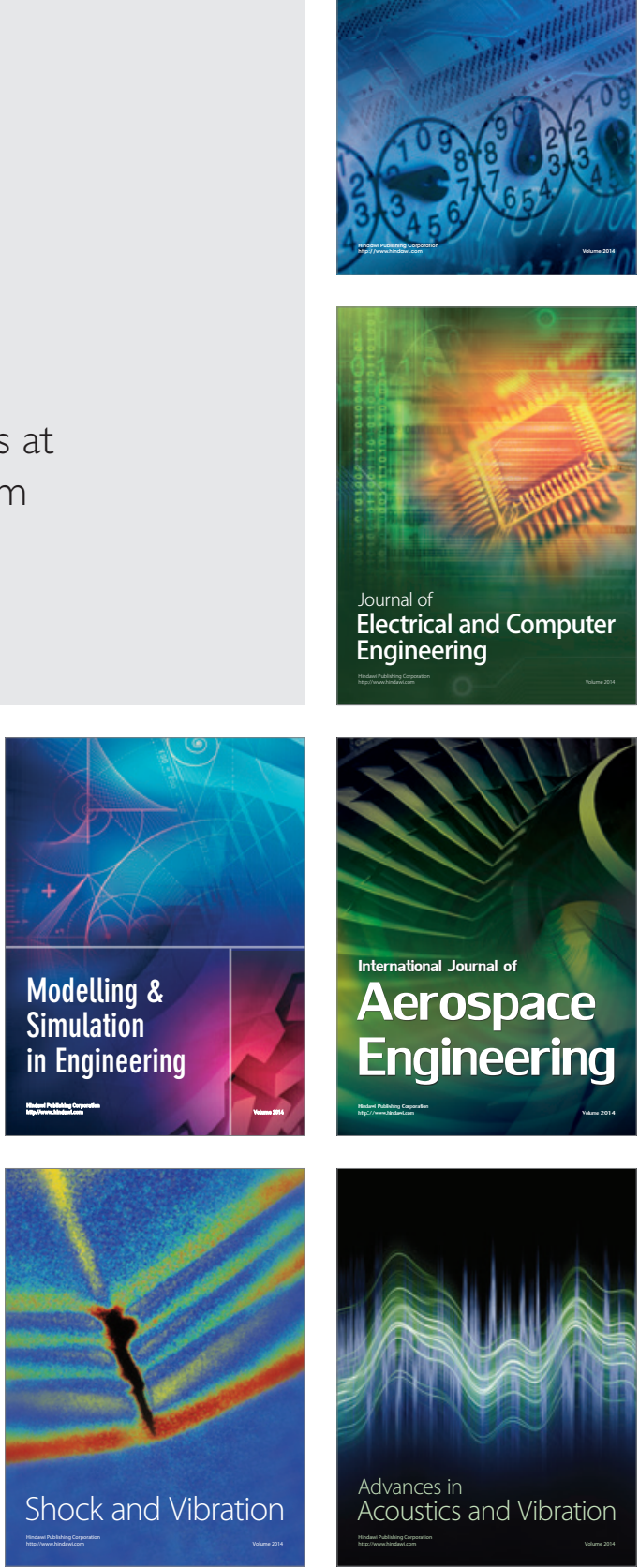